\title{
Distortional solutions for loaded semi-discretized thin-walled beams
}

\section{Andreassen, Michael Joachim; Jönsson, Jeppe}

\section{Published in:}

Thin-Walled Structures

Link to article, DOI:

10.1016/j.tws.2011.08.013

Publication date:

2012

Link back to DTU Orbit

Citation (APA):

Andreassen, M. J., \& Jönsson, J. (2012). Distortional solutions for loaded semi-discretized thin-walled beams. Thin-Walled Structures, 50(1), 116-127. https://doi.org/10.1016/j.tws.2011.08.013

\section{General rights}

Copyright and moral rights for the publications made accessible in the public portal are retained by the authors and/or other copyright owners and it is a condition of accessing publications that users recognise and abide by the legal requirements associated with these rights.

- Users may download and print one copy of any publication from the public portal for the purpose of private study or research.

- You may not further distribute the material or use it for any profit-making activity or commercial gain

- You may freely distribute the URL identifying the publication in the public portal

If you believe that this document breaches copyright please contact us providing details, and we will remove access to the work immediately and investigate your claim. 


\title{
Distortional solutions for loaded semi-discretized thin-walled beams
}

\author{
M. J. Andreassen, J. Jönsson \\ Technical University of Denmark, Department of Civil Engineering, Brovej Building 118, \\ DK-2800 Kgs. Lyngby
}

\begin{abstract}
For thin-walled beams, the classic theory for flexural and torsional analysis of open and closed cross-sections can be generalized by including distortional displacements. In a companion paper it is shown, that using a novel semidiscretization process, it is possible to determine specific distortional displacement fields which decouple the reduced order differential equations. In this process the cross section is discretized into finite cross-section elements, and the natural distortional modes as well as the related axial variations are found as solutions to the established coupled fourth order homogeneous differential equations of GBT.

In this paper the non-homogeneous distortional differential equations of GBT are formulated using this novel semidiscretization process. Transforming these non-homogeneous distortional differential equations into the natural eigenmode space by using the distortional modal matrix found for the homogeneous system, we get the uncoupled set of differential equations including the distributed loads. This uncoupling is very important in GBT, since the shear stiffness contribution from St. Venant torsional shear stress as well as "Bredt's shear flow" cannot be neglected nor approximated by the combination of axial stiffness and transverse stiffness, especially for closed cross sections. The full analytical solutions of these linear non-homogeneous differential equations are given, including four illustrative examples, which illustrate the strength of this novel approach to GBT. This new approach is a considerable theoretical achievement, since it without approximation gives the full analytical solution for a given discretization of the cross section including distributed loading. The boundary conditions considered in the examples of this paper are restricted to built in ends, which are needed for future displacement formulation of an exact first-order order distortional beam element.
\end{abstract}

Key words: Distortion, Warping, Distortional beam theory, Generalized beam theory (GBT), Thin-walled beams, Beam theory, Semi-discretization, Non-Homogeneous, Load.

\section{Introduction}

Thin-walled members are often used in the civil, mechanical and aerospace industry because of the high strength and the effective use of material. Due to the increased consumption of thin-walled structural elements there has been increasing focus and need for more detailed calculations. Thus, it has been necessary to extend the classic beam theory to include the distortion of the cross section. Such an extension of the theory is considered in this paper and in the companion paper [1] where a novel approach to the determination of distortional displacement modes of Generalized Beam Theory (GBT) is formulated. This novel approach involves a

Email addresses: mican@byg.dtu.dk (M. J. Andreassen), jej@byg.dtu.dk (J. Jönsson)

Preprint submitted to Thin-Walled Structures new cross-section semi-discretization process as well as a novel determination of the natural cross-section eigenmodes and related axial solution functions by exact analytical solution of the related first-order GBT equations. A variety of other formulations including distortional displacements have been proposed for analysis of both open and closed cross sections. Specially, the traditional first generation of generalized beam theory, known as GBT, initially proposed by Schardt in 1966 [2], has been very popular and fostered a lot of research and developments, mostly undertaken by a few independently working European groups, among others by Schardt [3], Davies [4], Lepistö [5], Baláž and Rendek [6], Simões da Silva and Simão [7], Gonçalves et al [8], Gonçalves and Camotim [9] and Camotim \& Silvestre [10], [11]. In these developments the distortional modes of traditional GBT have been extended (with

August 23, 2011 
"other" modes) in order to encompass shear through shear modes, post buckling through inclusion of transverse extension modes as well special modes to accommodate shear lag. However, these extensions with other modes are not considered in the present paper, since they are not part of a first generation GBT and may be viewed as patches towards expanded use of a generalized beam theory in a finite element context. For a more elaborate introduction see the companion paper, [1]. Particularly relevant in relation to our research is the closely related work of Hanf [12] as well as the work on distortional theory of thin-walled beams by Jönsson [13]. In contrast to and as a considerable advance on the traditional GBT formulation this novel GBT approach solves the fourth-order differential equations to obtain the distortional displacements for a linear beam analysis. This advance will enable future formulation of exact distortional beam elements with distributed load for first-order analysis using the found axial solution functions (solutions of the GBT equations) instead of conventional interpolation by third-order polynomials.

Thus, in this paper the distortional differential equations including distributed loads are formulated. Transforming the non-homogeneous distortional differential equations into an eigenmode space, by using the distortional modal matrix found for the homogeneous system, we get the diagonalized and thus uncoupled set of differential equations including the distributed loads. The full solution of these uncoupled linear differential equations is given and followed by four illustrative examples. The boundary conditions considered in the examples of this paper are restricted to built in ends, which are needed for future displacement formulation of an exact first-order distortional beam element. It should be mentioned that the theory and formulations in paper [1] remain valid, which implies that mainly the development of the particular part and the following final general solution will be presented in this paper. It was found necessary to follow the individual steps of the transformations and eliminations in the companion paper [1], in order to ensure a correct formulation of the individual decoupled non-homogeneous differential equation, especially for the distortional modes where we have utilized reduction of the order of the differential equations. Having done this once we may use work or energy principles to identify the individual load terms in a more direct manner.

\section{Basic kinematic assumptions}

The theories of beams are derived on the basis of assumed displacement fields which correspond to extension, flexure, torsion, warping and distortional displace-
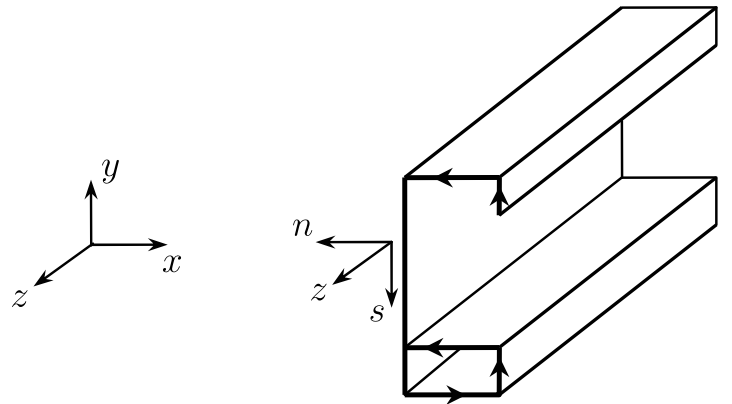

Figure 1: Global and local Cartesian reference frames.

ments. This corresponds to a modal separation in which each mode has a set of transverse and axial displacement fields that may be coupled. Each of these crosssection displacement fields is factorized in a displacement mode which is a function of the in-plane coordinates, multiplied by a function of the axial coordinate, which describes the axial variation of the mode.

In the following the prismatic beam is described in a global Cartesian $(x, y, z)$ coordinate system as shown in Figure 1. The figure introduces and shows the local coordinates $(z, n, s)$ corresponding to the axial, normal and tangential directions. In the local coordinate system the displacement components of one displacement mode $u_{n}, u_{s}$ and $u_{z}$ are given by the separated displacement functions as follows

$u_{n}(s, z)=\mathrm{w}_{n}(s) \psi(z)$
$u_{s}(n, s, z)=\left(\mathrm{w}_{s}(s)-n \mathrm{w}_{n, s}(s)\right) \psi(z)$
$u_{z}(n, s, z)=-\left(\Omega(s)+n \mathrm{w}_{n}(s)\right) \psi^{\prime}(z)$

where the local components are shown in Figure 2. The corresponding strains become

$$
\begin{aligned}
& \varepsilon_{z}=-\left(\Omega+n \mathrm{w}_{n}\right) \psi^{\prime \prime} \\
& \varepsilon_{s}=\left(\mathrm{w}_{s, s}-n \mathrm{w}_{n, s s}\right) \psi \\
& \gamma=\gamma_{z s}=u_{z, s}+u_{s, z}=\left(\mathrm{w}_{s}-\Omega,_{s}-2 n \mathrm{w}_{n, s}\right) \psi^{\prime}
\end{aligned}
$$

These are described in greater detail in the companion paper [1].

\section{Energy Assumptions}

The internal energy potential introduced in paper [1] will be briefly presented in this section followed by a separate introduction of the external energy potential for distributed loads. 


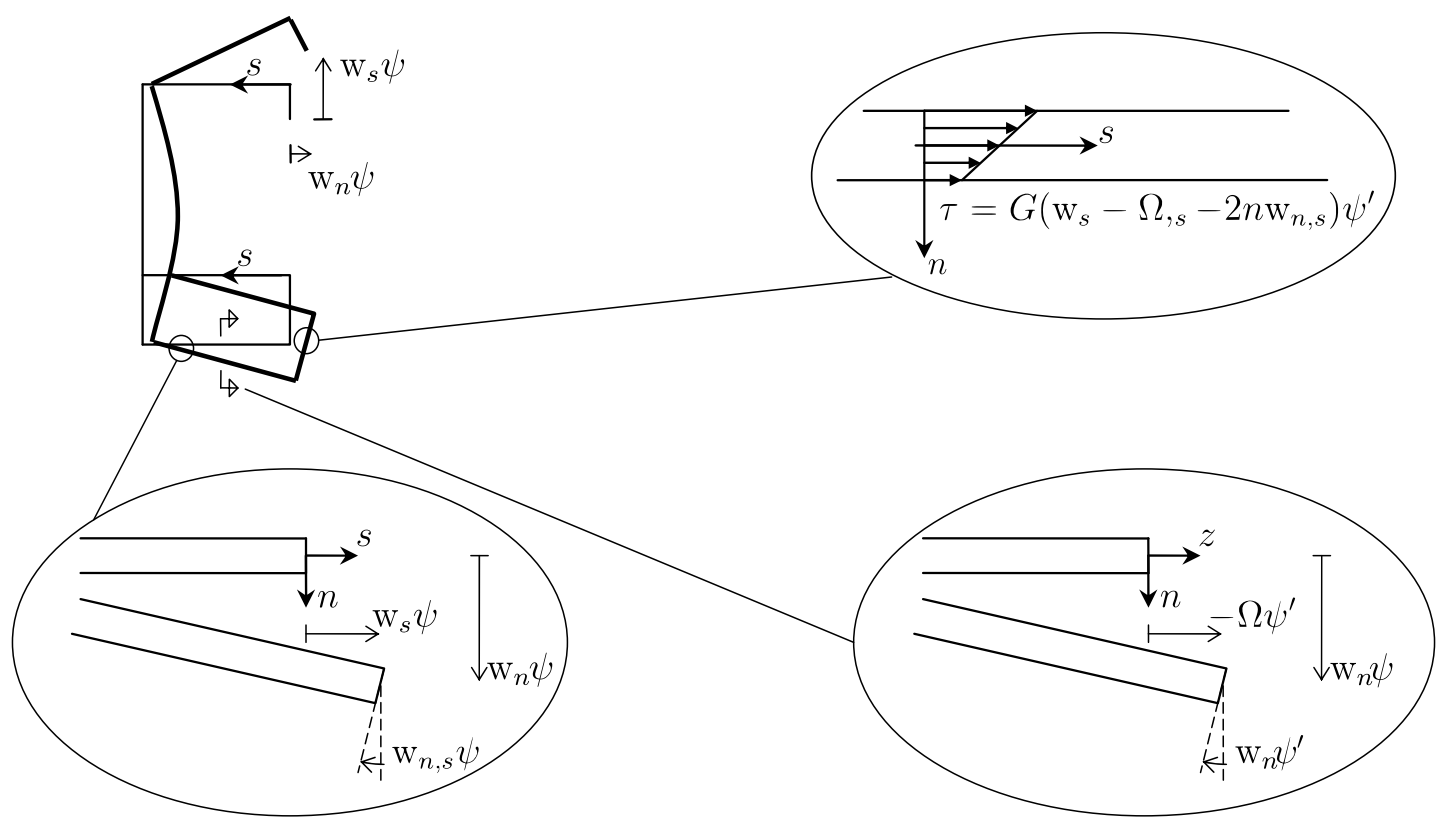

Figure 2: Local components of displacements and assumed shear stresses.

\subsection{Internal energy potential}

In the classic beam theory simple constitutive relations are used, which means that the material is assumed to be linear elastic with a modulus of elasticity $E$ and shear modulus $G$. In this paper also a plate elasticity modulus $E_{s}=E /\left(1-v^{2}\right)$ in the transverse direction will be utilized. The axial stress is determined as $\sigma_{z}=E \varepsilon_{z}$, the shear stress as $\tau=G \gamma$ and finally the transverse stress as $\sigma_{s}=E_{s} \varepsilon_{s}$. Thus taking the transverse plate bending effect into account but neglecting the coupling of axial strain $\varepsilon_{z}$ and transverse strain $\varepsilon_{s}$. With the constitutive relations assumed the elastic energy potential becomes

$\Pi_{i n t}=\int_{V}\left(\frac{1}{2} E \varepsilon_{z}^{2}+\frac{1}{2} G \gamma^{2}+\frac{1}{2} E_{s} \varepsilon_{s}^{2}\right) d V$

Let us introduce a thin-walled cross section assembled by using straight cross sectional elements. This allows us to integrate the internal energy across the volume of the thin-walled beam. In the following we will denote the thickness of the individual plane cross section elements by $t$ and the width by $b_{e l}$. The elastic potential energy of one mode takes the following form after the introduction of the strains expressed by the separated displacement functions:

$$
\begin{aligned}
& \Pi_{\text {int }}= \\
& \begin{aligned}
& \frac{1}{2} \int_{0}^{L}\left[\sum _ { e l } \int _ { 0 } ^ { b _ { e l } } \left\{\left[E t\left(\Omega \psi^{\prime \prime}\right)^{2}+\frac{1}{12} E t^{3}\left(\mathrm{w}_{n} \psi^{\prime \prime}\right)^{2}\right]\right.\right. \\
&+ {\left[G t\left(\mathrm{w}_{s} \psi^{\prime}\right)^{2}+G t\left(\Omega,{ }_{s} \psi^{\prime}\right)^{2}\right.} \\
&\left.\quad-2 G t\left(\mathrm{w}_{s} \psi^{\prime}\right)\left(\Omega,{ }_{s} \psi^{\prime}\right)+\frac{1}{3} G t^{3}\left(\mathrm{w}_{n, s} \psi^{\prime}\right)^{2}\right] \\
&\left.\left.+\left[E_{s} t\left(\mathrm{w}_{s, s} \psi\right)^{2}+\frac{1}{12} E_{s} t^{3}\left(\mathrm{w}_{n, s s} \psi\right)^{2}\right]\right\} d s\right] d z
\end{aligned}
\end{aligned}
$$

The elastic energy terms have been grouped in axial strain energy, shear energy, and transverse strain energy. Introducing the displacement interpolation functions leads to the definition of several stiffness submatrices as given in Table 1. The superscripts $\sigma, \tau$ and $s$ correspond to components of the axial stiffness, shear stiffness and transverse stiffness, respectively.

\subsection{External energy potential for distributed loads}

Let us now introduce three types of distributed loads $q_{z}, q_{s}, q_{n}$ which act on the mid plane of the individual walls in the $z, s, n$ directions, respectively. The external load potential for these distributed loads can then for one mode be found as

$\Pi_{\text {ext }}=-\int_{0}^{L} \int_{0}^{b_{e l}}\left[q_{z} u_{z}+q_{s} u_{s}+q_{n} u_{n}\right] d s d z$ 

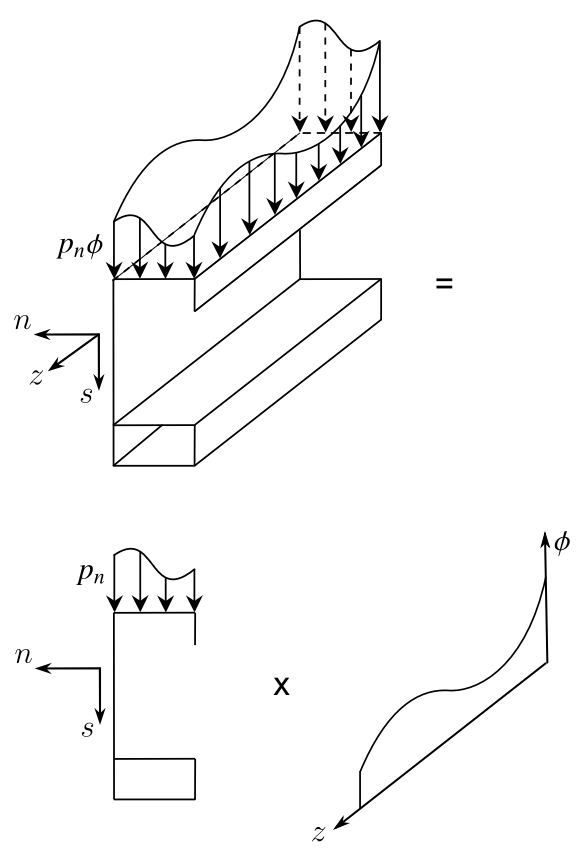

Figure 3: Load distribution.

Using separation of variables for the distributed loads as for the displacements, we introduce the following load variables $q_{s}=p_{s}(s) \phi(z), q_{n}=p_{n}(s) \phi(z), q_{z}=p_{z}(s) \phi(z)$. In this formulation $p_{s}, p_{n}, p_{z}$ represent the cross-section load distribution, and the function $\phi$ represents the axial variation of the loads. In the following formulation we operate with only one cross-section load distribution, which may be modified by summation of various different cross section load distributions and axial load variation functions. The load separation is illustrated in Figure 3 for a distributed load $q_{n}=p_{n}(s) \phi(z)$ on the upper flange of a thin-walled beam. The local components of the loads and force vectors for a cross section wall element is shown in Figure 4. Hereby the contribution to the external load potential of a single wall element takes the following form

$$
\begin{aligned}
\Pi_{e x t, e l}=-\int_{0}^{L} \int_{0}^{b_{e l}} \phi\left[p_{s} w_{s} \psi\right. & +p_{n} w_{n} \psi \\
& \left.-p_{z} \Omega \psi^{\prime}\right] d s d z
\end{aligned}
$$

which is suited for adequate interpolation in the following. Note that the two first load terms perform work through the transverse displacements and the last load term performs work through the axial warping displacements. Since the formulation of distortion has much in common with torsion the first two terms may be described as distortional moment loads and the last term as distortional bimoment load, see [13]. For the classic torsional equilibrium equation including warping of the cross section, see [14], these loads correspond to torsional moment load and torsional bimoment load.

\section{Interpolation within cross-section elements}

The interpolations related to the cross section are the displacement interpolations for $w_{s}, w_{n}$ and $\Omega$ described in the companion paper [1], and the interpolation of the cross section loads $p_{s}, p_{n}$ and $p_{z}$ introduced in the following. The distributed load shown in Figure 3 will be defined by a linear interpolation of the load on each cross section wall element multiplied by an axial shape function $\phi(z)$, for which we will introduce a specific interpolation later in a following section. The load interpolation in a cross-section wall is given by

$p_{s}=\mathbf{N}_{p} \mathbf{p}_{s}^{e l}, \quad p_{n}=\mathbf{N}_{p} \mathbf{p}_{n}^{e l}, \quad p_{z}=\mathbf{N}_{p} \mathbf{p}_{z}^{e l}$

in which $\mathbf{N}_{p}(s)=\left[1-s / b_{e l}, s / b_{e l}\right]$ is the linear interpolation matrix, and where the nodal end values of a cross-section wall element are given as

$\mathbf{p}_{s}^{e l}=\left[\begin{array}{c}p_{s 1} \\ p_{s 2}\end{array}\right], \quad \mathbf{p}_{n}^{e l}=\left[\begin{array}{l}p_{n 1} \\ p_{n 2}\end{array}\right], \quad \mathbf{p}_{z}^{e l}=\left[\begin{array}{c}p_{z 1} \\ p_{z 2}\end{array}\right]$

Using the introduced interpolations for the displacements and the loads, the external potential energy now takes the following form for a single wall element

$$
\begin{aligned}
& \Pi_{\text {ext }, e l}= \\
& -\int_{0}^{L} \int_{0}^{b_{e l}}\left[\psi \mathbf{v}_{\mathrm{w}}^{e l} \mathbf{N}_{s}{ }^{T} \mathbf{N}_{p} \mathbf{p}_{s}^{e l}+\psi \mathbf{v}_{\mathrm{w}}^{e l} \mathbf{N}_{n}{ }^{T} \mathbf{N}_{p} \mathbf{p}_{n}^{e l}\right. \\
& \left.-\psi^{\prime} \mathbf{v}_{\Omega}^{e l}{ }^{T} \mathbf{N}_{\Omega}{ }^{T} \mathbf{N}_{p} \mathbf{p}_{z}^{e l}\right] \phi d s d z
\end{aligned}
$$

This formulation allows us to write the element load vector in the same format as the element stiffness contributions from paper [1]. These are shown in Table 1 , where we have also included the nodal cross section wall loads $\mathbf{P}_{\mathrm{w}}^{e l}$ and $\mathbf{P}_{\Omega}^{e l}$ corresponding to line loads also varying along the beam with $\phi$. Hereby the walls of the thin-walled beam can be loaded by line loads acting at the cross section nodes, and by surface loads acting on the mid-plane of a cross section wall. Both of these loads are distributed along the beam as given by the $\phi$ function. Now we can rewrite the external load potential of a single wall element as,

$\Pi_{\text {ext }, e l}=-\int_{0}^{L}\left[\psi \mathbf{v}_{\mathrm{w}}^{e l} \mathbf{r}_{\mathrm{w}}^{e l} \phi-\psi^{\prime} \mathbf{v}_{\Omega}^{e l} \mathbf{r}_{\Omega}^{e l} \phi\right] d z$

where we have introduced the axial and transverse nodal load components of a straight cross-section element as,

$$
\begin{aligned}
& \mathbf{r}_{\Omega}^{e l}=\left[\begin{array}{ll}
r_{\Omega 1}^{e l} & r_{\Omega 2}^{e l}
\end{array}\right]^{T} \\
& \mathbf{r}_{\mathrm{w}}^{e l}=\left[\begin{array}{llllll}
r_{\mathrm{w} 1}^{e l} & r_{\mathrm{w} 2}^{e l} & r_{\mathrm{w} 3}^{e l} & r_{\mathrm{w} 4}^{e l} & r_{\mathrm{w} 5}^{e l} & r_{\mathrm{w} 6}^{e l}
\end{array}\right]^{T}
\end{aligned}
$$




$$
\begin{aligned}
& \mathbf{k}_{\Omega \Omega}^{\sigma}=\int_{0}^{b_{e}} E t \mathbf{N}_{\Omega}^{T} \mathbf{N}_{\Omega} d s \\
& \mathbf{k}_{\mathrm{ww}}^{\sigma}=\int_{0}^{b_{e}} \frac{E t^{3}}{12} \mathbf{N}_{n}^{T} \mathbf{N}_{n} d s \\
& \mathbf{k}^{s}=\int_{0}^{b_{e}}\left(E_{s} t \mathbf{N}_{s, s}^{T} \mathbf{N}_{s, s}+\frac{E_{s} t^{3}}{12} \mathbf{N}_{n, s s}^{T} \mathbf{N}_{n, s s}\right) d s \\
& \mathbf{k}_{\mathrm{ww}}^{\tau}=\int_{0}^{b_{e}}\left(G t \mathbf{N}_{s}^{T} \mathbf{N}_{s}+\frac{G t^{3}}{3} \mathbf{N}_{n, s}^{T} \mathbf{N}_{n, s}\right) d s \\
& \mathbf{k}_{\Omega \Omega}^{\tau}=\int_{0}^{b_{e}} G t \mathbf{N}_{\Omega, s}^{T} \mathbf{N}_{\Omega, s} d s \\
& \mathbf{k}_{\mathrm{w} \Omega}^{\tau}=\left[\mathbf{k}_{\Omega \mathrm{w}}^{\tau}\right]^{T}=-\int_{0}^{b_{e}} G t \mathbf{N}_{s}^{T} \mathbf{N}_{\Omega, s} d s \\
& \mathbf{r}_{\Omega}^{e l}=\int_{0}^{b_{e}} \mathbf{N}_{\Omega}^{T} \mathbf{N}_{p} d s \mathbf{p}_{z}^{e l}+\mathbf{P}_{\Omega}^{e l} \\
& \mathbf{r}_{\mathrm{w}}^{e l}=\int_{0}^{b_{e}} \mathbf{N}_{s}^{T} \mathbf{N}_{p} d s \mathbf{p}_{s}^{e l}+\int_{0}^{b_{e}} \mathbf{N}_{n}^{T} \mathbf{N}_{p} d s \mathbf{p}_{n}^{e l}+\mathbf{P}_{\mathrm{w}}^{e l}
\end{aligned}
$$

Table 1: Straight-element stiffness and load contributions.

These components are shown in Figure 4 along with the direction of the wall element coordinates $(n, s)$ as well as the positive direction of the load components. We choose to assemble the single element components into two separate global vectors containing the axial load and the transverse load, respectively. These global vectors we will write as follows:

$$
\begin{aligned}
& \mathbf{r}_{\Omega}=\left[\begin{array}{lllllll}
r_{\Omega 1} & r_{\Omega 2} & r_{\Omega 3} & \ldots
\end{array}\right]^{T} \\
& \mathbf{r}_{\mathrm{w}}=\left[\begin{array}{lllllll}
r_{\mathrm{w} 1} & r_{\mathrm{w} 2} & r_{\mathrm{w} 3} & r_{\mathrm{w} 4} & r_{\mathrm{w} 5} & r_{\mathrm{w} 6} & \ldots
\end{array}\right]^{T}
\end{aligned}
$$

where the transformation from local to global components is performed using a formal standard transformation of the components in the cross-section plane, i.e.

$$
\begin{aligned}
& \mathbf{r}_{\Omega}=\sum_{e l} \mathbf{T}_{\Omega}{ }^{T} \mathbf{r}_{\Omega}^{e l} \\
& \mathbf{r}_{\mathrm{w}}=\sum_{e l} \mathbf{T}_{\mathrm{w}}{ }^{T}{ }^{e l}{ }_{\mathrm{w}}^{e l}
\end{aligned}
$$

See Table 2 for a overview of the important transformations used in this and in the companion paper [1]. Now we can write the total potential energy by summation of each element contribution as

$\Pi_{t o t}=\Pi_{i n t}+\Pi_{e x t}, \quad$ where $\Pi_{e x t}=\sum_{e l} \Pi_{e x t, e l}$

where $\Pi_{\text {int }}$ is the contribution to the potential energy from the internal properties found in paper [1], and $\Pi_{\text {ext }}$ is the contribution from the external loads. Introducing the described interpolation and matrix calculation scheme allows us to write the total potential energy as

$$
\Pi_{t o t}=\Pi_{i n t}-\int_{0}^{L}\left\{\left(\psi \mathbf{v}_{\mathrm{w}}^{T}\right) \mathbf{r}_{\mathrm{w}} \phi-\left(\psi \mathbf{v}_{\Omega}^{T}\right)^{\prime} \mathbf{r}_{\Omega} \phi\right\} d z
$$
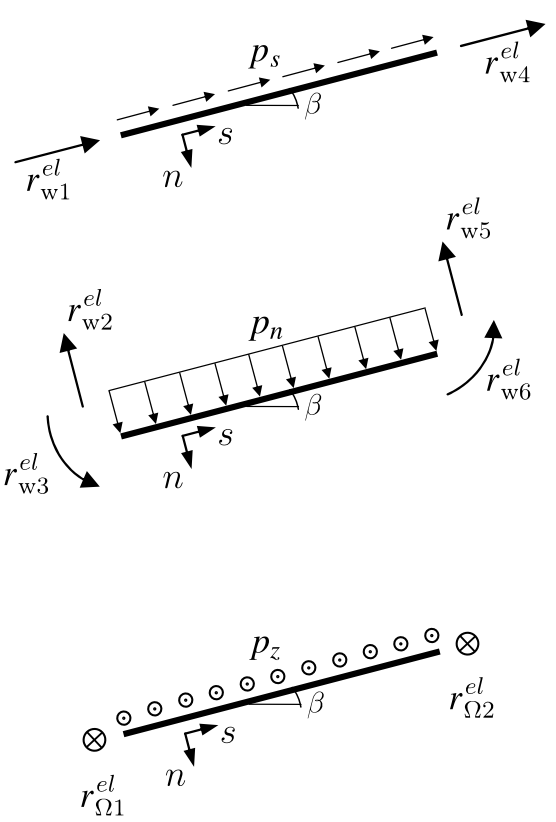

Figure 4: Distributed loads and the resulting load vectors.

The first term corresponds to the distortional moment load which performs work through the transverse displacements. The second term corresponds to the distortional bimoment load which performes work through the axial displacements.

\section{Modal loads and modal solutions}

To obtain a formulation resembling the generalization of Vlasov beam theory including distortion, the following three main steps are performed as in the companion paper [1]. This allows us to properly identify modal load components as well as the contributions to the individual modal differential equations.

\subsection{Step I - Pure axial load and shear constraints}

Following the procedure which is used to identify pure axial extension as an eigenmode and to introduce shear constraints, we will identify the axial load components and separate these from the remaining equations. The potential energy formulation including the load terms in equation (22) have to be modified, so that the pure axial extension is described by the separate degree of freedom $v_{\Omega}^{a}$, and so that the shear constraint equations are enforced. This modification is performed using the following transformation No. 5 described in Table 2:

$\mathbf{v}_{\Omega}=\mathbf{T}_{\Omega \mathrm{W}}^{r} \mathbf{v}_{\mathrm{W}}+\mathbf{T}_{\Omega}^{a} v_{\Omega}^{a}$ 


\begin{tabular}{|c|c|c|}
\hline No. & Description & Transformations \\
\hline 1 & Transformation from local to global axial d.o.f. & $\mathbf{v}_{\Omega}=\mathbf{T}_{\Omega} \mathbf{v}_{\Omega}^{e l}$ \\
\hline 2 & Transformation from local to global transverse d.o.f. & $\mathbf{v}_{\mathrm{w}}=\mathbf{T}_{\mathrm{w}} \mathbf{v}_{\mathrm{w}}^{e l}$ \\
\hline 3 & $\begin{array}{l}\text { Transformation from pure axial extension and other axial } \\
\text { d.o.f. to global axial d.o.f. }\end{array}$ & $\mathbf{v}_{\Omega}=\left[\begin{array}{ll}\mathbf{T}_{\Omega}^{a} & \mathbf{T}_{\Omega}^{o}\end{array}\right]\left[\begin{array}{l}\mathbf{v}_{\Omega}^{a} \\
\mathbf{v}_{\Omega}^{o}\end{array}\right]$ \\
\hline 4 & $\begin{array}{l}\text { Transformation from transverse d.o.f. to the other axial d.o.f. } \\
\text { (without pure axial extension) based on the shear constrains }\end{array}$ & $\mathbf{v}_{\Omega}^{o}=\mathbf{T}_{\Omega \mathrm{W}} \mathbf{v}_{\mathrm{W}}$ \\
\hline 5 & $\begin{array}{l}\text { Transformation from transverse d.o.f. and pure axial exten- } \\
\text { sion d.o.f. to the global axial d.o.f. }\end{array}$ & $\mathbf{v}_{\Omega}=\left[\begin{array}{ll}\mathbf{T}_{\Omega \mathrm{W}}^{r} & \mathbf{T}_{\Omega}^{a}\end{array}\right]\left[\begin{array}{l}\mathbf{v}_{\mathrm{W}} \\
v_{\Omega}^{a}\end{array}\right]$ \\
\hline 6 & $\begin{array}{l}\text { Transformation from pure transverse translation d.o.f., pure } \\
\text { rotation d.o.f., constant wall-width constrained d.o.f. and un- } \\
\text { constrained d.o.f. to global transverse d.o.f. (wall-width con- } \\
\text { straints not applied) }\end{array}$ & $\mathbf{v}_{\mathrm{w}}=\left[\begin{array}{llll}\mathbf{T}_{\mathrm{w}}^{\alpha} & \mathbf{T}_{\mathrm{w}}^{3} & \mathbf{T}_{\mathrm{w}}^{c} & \mathbf{T}_{\mathrm{w}}^{u}\end{array}\right]\left[\begin{array}{c}\mathbf{v}_{\mathrm{w}}^{\alpha} \\
v_{\mathrm{w}}^{3} \\
\mathbf{v}_{\mathrm{W}}^{c} \\
\mathbf{v}_{\mathrm{w}}^{u}\end{array}\right.$ \\
\hline 7 & $\begin{array}{l}\text { Transformation from pure transverse translation d.o.f., pure } \\
\text { rotation d.o.f. and unconstrained d.o.f. to global transverse } \\
\text { d.o.f. (wall-width constraints applied) }\end{array}$ & $\mathbf{v}_{\mathrm{w}}=\left[\begin{array}{lll}\mathbf{T}_{\mathrm{w}}^{\alpha} & \mathbf{T}_{\mathrm{w}}^{3} & \widetilde{\mathbf{T}}_{\mathrm{w}}^{u}\end{array}\right]\left[\begin{array}{c}\mathbf{v}_{\mathrm{w}}^{\alpha} \\
v_{\mathrm{w}}^{3} \\
\mathbf{v}_{\mathrm{w}}^{u}\end{array}\right]$ \\
\hline 8 & $\begin{array}{l}\text { Transformation from constrained transverse d.o.f. and trans- } \\
\text { verse GBT d.o.f. to global transverse d.o.f. }\end{array}$ & $\mathbf{v}_{\mathrm{w}}=\left[\begin{array}{ll}\mathbf{T}_{\mathrm{w}}^{c} & \mathbf{T}_{\mathrm{w}}^{g}\end{array}\right]\left[\begin{array}{c}\mathbf{v}_{\mathrm{W}}^{c} \\
\mathbf{v}_{\mathrm{w}}^{g}\end{array}\right]$ \\
\hline 9 & Transformation from FE space to GBT space & {$\left[\begin{array}{c}\mathbf{v}_{\mathrm{W}}^{g} \\
v_{\Omega}^{a}\end{array}\right]=\left[\begin{array}{cc}\mathbf{T}_{\mathrm{W}}^{g T} & \mathbf{0} \\
\mathbf{0} & \mathbf{T}_{\Omega}^{a T}\end{array}\right]\left[\begin{array}{l}\mathbf{v}_{\mathrm{W}} \\
\mathbf{v}_{\Omega}\end{array}\right]$} \\
\hline 10 & Transformation from GBT space to FE space & {$\left[\begin{array}{c}\mathbf{v}_{\mathrm{w}} \\
\mathbf{v}_{\Omega}\end{array}\right]=\left[\begin{array}{cc}\widetilde{\mathbf{T}}_{\mathrm{W}}^{g} & \mathbf{0} \\
\mathbf{T}_{\Omega \mathrm{w}}^{r} \widetilde{T}_{\mathrm{w}}^{g} & \mathbf{T}_{\Omega}^{a T}\end{array}\right]\left[\begin{array}{l}\mathbf{v}_{\mathrm{w}}^{g} \\
v_{\Omega}^{a}\end{array}\right]$} \\
\hline
\end{tabular}

Table 2: Transformations 


$$
r_{\Omega}^{a}=\mathbf{T}_{\Omega}^{a T} \mathbf{r}_{\Omega} \quad \overline{\mathbf{r}}_{\Omega}=\mathbf{T}_{\Omega \mathrm{w}}^{r}{ }^{T} \mathbf{r}_{\Omega}-\mathbf{K}_{\Omega \Omega}^{\sigma r a}\left(K_{\Omega \Omega}^{\sigma a a}\right)^{-1} r_{\Omega}^{a}
$$

Table 3: Transformation of load vectors related to Step I.

To clarify the variational treatment of pure axial extension, we also temporally rewrite the terms pertaining to axial extension using $\zeta v_{\Omega}^{a}=-\psi^{\prime} v_{\Omega}^{a}$. The modified elastic potential energy (for a single mode) takes the following form:

$\Pi_{t o t}=\Pi_{i n t}-$

$\int_{0}^{L}\left\{\left(\psi \mathbf{v}_{\mathrm{w}}^{T}\right) \mathbf{r}_{\mathrm{w}} \phi-\left(\psi \mathbf{v}_{\mathrm{w}}^{T}\right)^{\prime} \mathbf{T}_{\Omega \mathrm{w}}{ }^{T} \mathbf{r}_{\Omega} \phi+\left(\zeta v_{\Omega}^{a}\right) r_{\Omega}^{a} \phi\right\} d z$

in which the pure axial loading is identified as $r_{\Omega}^{a}$. It is as given in Table 3 identified as the product of the transpose of the pure axial deformation mode and the global axial load vector .

To obtain the differential equations of GBT, the first variation of the elastic potential energy is investigated by taking variations in the complete displacement field. This gives

$\delta \Pi_{t o t}=\delta \Pi_{i n t}-$

$\int_{0}^{L}\left\{\delta\left(\psi \mathbf{v}_{\mathrm{w}}^{T}\right) \mathbf{r}_{\mathrm{w}} \phi-\delta\left(\psi \mathbf{v}_{\mathrm{w}}^{T}\right)^{\prime} \mathbf{T}_{\Omega \mathrm{w}}^{r}{ }^{T} \mathbf{r}_{\Omega} \phi+\delta\left(\zeta v_{\Omega}^{a}\right) r_{\Omega}^{a} \phi\right\} d z$

After performing partial integrations on the terms that involve axial derivatives of the (virtual) varied displacement field, $\delta()^{\prime}$, the first variation of the elastic potential energy takes the form

$$
\begin{aligned}
& \delta \Pi_{\text {tot }}=\delta \Pi_{\text {int }}- \\
& \int_{0}^{L}\left\{\delta\left(\psi \mathbf{v}_{\mathrm{w}}^{T}\right)\left[\mathbf{r}_{\mathrm{w}} \phi+\mathbf{T}_{\Omega \mathrm{w}}^{r}{ }^{T} \mathbf{r}_{\Omega} \phi^{\prime}\right]+\delta\left(\zeta v_{\Omega}^{a}\right) r_{\Omega}^{a} \phi\right\} d s \\
& +\left[\delta\left(\psi \mathbf{v}_{\mathrm{w}}^{T}\right)\left[\mathbf{T}_{\Omega \mathrm{w}}^{r}{ }^{T} \mathbf{r}_{\Omega} \phi\right]\right]_{0}^{L}
\end{aligned}
$$

For internal variation in the displacement fields $\delta\left(\psi \mathbf{v}_{\mathrm{w}}\right)$ and $\delta\left(\zeta v_{\Omega}^{a}\right)$, the elastic potential energy should be stationary and therefore its first variation must be equal to zero. Here the terms in the squared bracket correspond to the boundary loads and boundary conditions. Substituting $\delta \Pi_{\text {int }}$ from the companion paper [1] leads to the following coupled non-homogeneous differential equations of GBT in which we note that $\zeta=-\psi^{\prime}$ :

$$
\begin{gathered}
\overline{\mathbf{K}}^{\sigma} \mathbf{v}_{\mathrm{w}} \psi^{\prime \prime \prime \prime}-\mathbf{K}_{\Omega \Omega}^{\sigma r a} v_{\Omega}^{a} \zeta^{\prime \prime \prime}-\mathbf{K}^{\tau} \mathbf{v}_{\mathrm{w}} \psi^{\prime \prime}+\mathbf{K}^{s} \mathbf{v}_{\mathrm{w}} \psi \\
=\mathbf{r}_{\mathrm{w}} \phi+\mathbf{T}_{\Omega \mathrm{w}}^{r}{ }^{T} \mathbf{r}_{\Omega} \phi^{\prime} \\
\mathbf{K}_{\Omega \Omega}^{\sigma a r} \mathbf{v}_{\mathrm{w}} \psi^{\prime \prime \prime}-K_{\Omega \Omega}^{\sigma a a} v_{\Omega}^{a} \zeta^{\prime \prime}=r_{\Omega}^{a} \phi
\end{gathered}
$$

Here the left hand side of the equations corresponds to the homogeneous equations, and the right hand side are the non homogeneous (load) terms. The stiffness matrices, $\mathbf{K}$, are found and described in paper [1].

These equations establish a coupled set of nonhomogeneous GBT differential equations, that determine the displacements of a thin-walled beam for a given set of boundary conditions. The homogeneous parts of the solution have been found, and now we seek particular solutions to the modal equations. Let us start out by isolating the term $v_{\Omega}^{a} \zeta^{\prime \prime}$ in equation (28) as

$v_{\Omega}^{a} \zeta^{\prime \prime}=\left(K_{\Omega \Omega}^{\sigma a a}\right)^{-1}\left[\mathbf{K}_{\Omega \Omega}^{\sigma a r} \mathbf{v}_{\mathbf{w}} \psi^{\prime \prime \prime}-r_{\Omega}^{a} \phi\right]$

Let us then first consider the pure axial extension mode, which has been identified as $\left(\mathbf{v}_{\mathrm{w}}, v_{\Omega}^{a}\right)=(\mathbf{0}, 1)$, where we introduce the notation bold zero $\mathbf{0}$ for a suitable size matrix or vector of zeroes. Introducing this mode in equation (29) uncouples the equation (since $\mathbf{v}_{\mathrm{w}}=\mathbf{0}$ ). Integrating the particular solution for the axial mode, the complete solution for the axial variation is then given by adding the homogeneous part of the solution and the particular part as follows:

$\zeta(z)=\left[\begin{array}{ll}1 & z\end{array}\right]\left[\begin{array}{l}c_{a 1} \\ c_{a 2}\end{array}\right]-\left(K_{\Omega \Omega}^{\sigma a a}\right)^{-1} r_{\Omega}^{a} \iint \phi d z d z$

where $c_{a 1}$ and $c_{a 2}$ are constants determined by the boundary conditions of axial extension.

In the context of the current work we will interpolate the cross section load using one distribution function $\phi(z)$, which varies linearly between two end values $\left(\phi_{1}\right.$ and $\left.\phi_{2}\right)$ representing the values of the multiplicative function at the ends of the profile. Thus we introduce $\phi$ as

$\phi=\left[\begin{array}{ll}1-\frac{z}{L} & z \\ L\end{array}\right] \boldsymbol{\phi}$, where $\boldsymbol{\phi}=\left[\begin{array}{l}\phi_{1} \\ \phi_{2}\end{array}\right]$

Using this linear interpolation the full integrated solution of equation (30) takes the form

$\zeta(z)=-\boldsymbol{\Psi}_{a h^{\prime}}{ }^{\prime}(z) \mathbf{c}_{a}-\boldsymbol{\Psi}_{a p}{ }^{\prime}(z) \boldsymbol{\phi}$

where

$\boldsymbol{\Psi}_{a h^{\prime}}{ }^{\prime}=-\left[\begin{array}{ll}1 & z\end{array}\right] \quad \mathbf{c}_{a}=\left[\begin{array}{l}c_{a 1} \\ c_{a 2}\end{array}\right]$
$\boldsymbol{\Psi}_{a p}{ }^{\prime}=\frac{L^{2} r_{\Omega}^{a}}{6 K_{\Omega \Omega}^{\text {Gaa }}}\left[3\left(\frac{z}{L}\right)^{2}-\left(\frac{z}{L}\right)^{3} \quad\left(\frac{z}{L}\right)^{3}\right]$

The introduced subscripts $h$ and $p$ denote the homogeneous and the particular parts of the solution, respectively.

Next let us consider the formulation of the remaining transverse displacement modes. Inserting equation 


$$
\begin{array}{ll}
\mathbf{r}_{\mathrm{w}}^{\alpha}=\mathbf{T}_{\mathrm{w}}^{\alpha T} \mathbf{r}_{\mathrm{w}} & r_{\mathrm{w}}^{3}=\mathbf{T}_{\mathrm{w}}^{3 T} \mathbf{r}_{\mathrm{w}} \\
\mathbf{r}_{\mathrm{w}}^{u}=\widetilde{\mathbf{T}}_{\mathrm{w}}^{u T} \mathbf{r}_{\mathrm{w}} & \mathbf{r}_{\mathrm{w}}^{u \alpha}=\mathbf{r}_{\mathrm{w}}^{u}-\mathbf{K}_{\alpha u}^{\sigma T}\left(\mathbf{K}_{\alpha \alpha}^{\sigma}\right)^{-1} \mathbf{r}_{\mathrm{w}}^{\alpha} \\
\overline{\mathbf{r}}_{\Omega}^{\alpha}=\mathbf{T}_{\mathrm{w}}^{\alpha T} \overline{\mathbf{r}}_{\Omega} & \bar{r}_{\Omega}^{3}=\mathbf{T}_{\mathrm{w}}^{3 T} \overline{\mathbf{r}}_{\Omega} \\
\overline{\mathbf{r}}_{\Omega}^{u}=\widetilde{\mathbf{T}}_{\mathrm{w}}^{u T} \overline{\mathbf{r}}_{\Omega} & \overline{\mathbf{r}}_{\Omega}^{u \alpha}=\overline{\mathbf{r}}_{\Omega}^{u}-\mathbf{K}_{\alpha u}^{\sigma T}\left(\mathbf{K}_{\alpha \alpha}^{\sigma}\right)^{-1} \overline{\mathbf{r}}_{\Omega}^{\alpha}
\end{array}
$$

Table 4: Transformation of load vectors related to Step II.

(29) differentiated once into equation (27) we eliminate pure axial extension. Introducing $\mathbf{K}^{\sigma}$ as in paper [1], we obtain the following non-homogeneous fourth order differential equations for determination of the transverse (global, distortional and local) distortional displacement modes of GBT:

$$
\begin{aligned}
\mathbf{K}^{\sigma} \mathbf{v}_{\mathrm{w}} \psi^{\prime \prime \prime \prime}-\mathbf{K}^{\tau} \mathbf{v}_{\mathrm{w}} \psi^{\prime \prime}+\mathbf{K}^{s} \mathbf{v}_{\mathrm{w}} \psi \\
=\mathbf{r}_{\mathrm{w}} \phi+\left(\mathbf{T}_{\Omega \mathrm{w}}^{r}{ }^{T} \mathbf{r}_{\Omega}-\mathbf{K}_{\Omega \Omega}^{\sigma r a}\left(K_{\Omega \Omega}^{\sigma a a}\right)^{-1} r_{\Omega}^{a}\right) \phi^{\prime}
\end{aligned}
$$

which we choose to abbreviate and write as

$$
\mathbf{K}^{\sigma} \mathbf{v}_{\mathrm{w}} \psi^{\prime \prime \prime \prime}-\mathbf{K}^{\tau} \mathbf{v}_{\mathrm{w}} \psi^{\prime \prime}+\mathbf{K}^{s} \mathbf{v}_{\mathrm{w}} \psi=\mathbf{r}_{\mathrm{w}} \phi+\overline{\mathbf{r}}_{\Omega} \phi^{\prime}
$$

where $\overline{\mathbf{r}}_{\Omega}$ is given in Table 3 .

\subsection{Step II - Flexural loading and constant wall width}

In this step we treat two modes corresponding to transverse translations of the cross section, and one mode corresponding to pure rotation. We also constrain the transverse displacement field so that the wall widths remain constant, i.e. we enforce $\mathrm{w}_{s, s} \equiv 0$.

Let us do this by first introducing transformation No. 7 from Table 2 into the differential equations in (34), and also introduce the null terms corresponding to the rigid-body modes and zero shear strain for translational and flexural modes. Hereby the differential equations including the load terms take the following form:

$$
\begin{gathered}
{\left[\begin{array}{ccc}
\mathbf{K}_{\alpha \alpha}^{\sigma} & \mathbf{0} & \mathbf{K}_{\alpha u}^{\sigma} \\
\mathbf{0} & K_{33}^{\sigma} & \mathbf{K}_{3 u}^{\sigma} \\
\mathbf{K}_{u \alpha}^{\sigma} & \mathbf{K}_{u 3}^{\sigma} & \mathbf{K}_{u u}^{\sigma}
\end{array}\right]\left[\begin{array}{c}
\mathbf{v}_{\mathrm{w}}^{\alpha} \\
v_{\mathrm{w}}^{3} \\
\mathbf{v}_{\mathrm{w}}^{\mathbf{u}}
\end{array}\right] \psi^{\prime \prime \prime \prime}-\left[\begin{array}{ccc}
\mathbf{0} & \mathbf{0} & \mathbf{0} \\
\mathbf{0} & K_{33}^{\tau} & \mathbf{K}_{3 u}^{\tau} \\
\mathbf{0} & \mathbf{K}_{u 3}^{\tau} & \mathbf{K}_{u u}^{\tau}
\end{array}\right]\left[\begin{array}{c}
\mathbf{v}_{\mathrm{w}}^{\alpha} \\
v_{\mathrm{w}}^{3} \\
\mathbf{v}_{\mathrm{w}}^{\mathbf{u}}
\end{array}\right] \psi^{\prime \prime}} \\
+\left[\begin{array}{ccc}
\mathbf{0} & \mathbf{0} & \mathbf{0} \\
\mathbf{0} & 0 & \mathbf{0} \\
\mathbf{0} & \mathbf{0} & \mathbf{K}_{u u}^{s}
\end{array}\right]\left[\begin{array}{c}
\mathbf{v}_{\mathrm{w}}^{\alpha} \\
v_{\mathrm{w}}^{3} \\
\mathbf{v}_{\mathrm{w}}^{\mathbf{u}}
\end{array}\right] \psi=\left[\begin{array}{c}
\mathbf{r}_{\mathrm{w}}^{\alpha} \\
r_{\mathrm{w}}^{3} \\
\mathbf{r}_{\mathrm{w}}^{u}
\end{array}\right] \phi+\left[\begin{array}{c}
\overline{\mathbf{r}}_{\Omega}^{\alpha} \\
\bar{r}_{\Omega}^{3} \\
\overline{\mathbf{r}}_{\Omega}^{u}
\end{array}\right] \phi^{\prime}
\end{gathered}
$$

where the transformed stiffness matrices are found and described in paper [1] and the load vectors are given in table 4 . The two-dimensional upper block matrix equation yields the translation displacements as

$$
\mathbf{v}_{\mathrm{w}}^{\alpha} \psi^{\prime \prime \prime \prime}=\mathbf{K}_{\alpha \alpha}^{\sigma-1}\left[\mathbf{r}_{\mathrm{w}}^{\alpha} \phi+\overline{\mathbf{r}}_{\Omega}^{\alpha} \phi^{\prime}-\mathbf{K}_{\alpha u}^{\sigma} \mathbf{v}_{\mathrm{w}}^{u} \psi^{\prime \prime \prime \prime}\right]
$$

where $\alpha=1$ or $\alpha=2$. We can identify the two orthogonal pure translational modes, $\left(v_{\mathrm{w}}^{1}, v_{\mathrm{w}}^{2}, v_{\mathrm{w}}^{3}, \mathbf{v}_{\mathrm{w}}^{u}\right)=$ $(1,0,0, \mathbf{0})$ and $(0,1,0, \mathbf{0})$, as eigenmodes or full solutions to the homogeneous part of equation (35). A particular solution for the axial variation of the pure translational modes is determined by quadruple integration of the non homogeneous load terms ( complete solution is then given by summation of the full homogeneous solution and the particular solution, which we can express as

$$
\begin{aligned}
& \psi_{\alpha}(z)=c_{\alpha 1}+c_{\alpha 2} z+c_{\alpha 3} z^{2}+c_{\alpha 4} z^{3} \\
&+\iiint \int\left(\mathbf{K}_{\alpha \alpha}^{\sigma}\right)^{-1}\left(\mathbf{r}_{\mathrm{w}}^{\alpha} \phi+\overline{\mathbf{r}}_{\Omega}^{\alpha} \phi^{\prime}\right) d z d z d z d z
\end{aligned}
$$

Remembering that we in the present context introduce $\phi$ as one linear function as given in equation (31), we can perform the quadruple integration and get

$\psi_{\alpha}(z)=\boldsymbol{\Psi}_{\alpha h}(z) \mathbf{c}_{\alpha}+\boldsymbol{\Psi}_{\alpha p}(z) \boldsymbol{\phi}$

Here $\mathbf{c}_{\alpha}$ is a vector containing four constants of the homogeneous part of the solution, and

$$
\begin{aligned}
& \boldsymbol{\Psi}_{\alpha p}(z)=\boldsymbol{\Psi}_{\alpha p}^{\mathrm{w}}(z)+\boldsymbol{\Psi}_{\alpha p}^{\Omega}(z) \\
& \boldsymbol{\Psi}_{\alpha h}(z)=\left[\begin{array}{llll}
1 & z & z^{2} & z^{3}
\end{array}\right] \\
& \boldsymbol{\Psi}_{\alpha p}^{\mathrm{w}}(z)=\frac{L^{4}}{120}\left(\mathbf{K}_{\alpha \alpha}^{\sigma}\right)^{-1} \mathbf{r}_{\mathrm{w}}^{\alpha}\left[5\left(\frac{z}{L}\right)^{4}-\left(\frac{z}{L}\right)^{5} \quad\left(\frac{z}{L}\right)^{5}\right] \\
& \boldsymbol{\Psi}_{\alpha p}^{\Omega}(z)=\frac{L^{3}}{24}\left(\mathbf{K}_{\alpha \alpha}^{\sigma}\right)^{-1} \overline{\mathbf{r}}_{\Omega}^{\alpha}\left[4\left(\frac{z}{L}\right)^{3}-\left(\frac{z}{L}\right)^{4} \quad\left(\frac{z}{L}\right)^{4}\right]
\end{aligned}
$$

Having identified the solutions related to the two pure translational modes we return to the remaining block equations of equation (35). Eliminating the two pure flexural degrees of freedom using equation (36) we obtain the condensed version of the differential equation (35) as

$$
\begin{gathered}
{\left[\begin{array}{cc}
K_{33}^{\sigma} & \mathbf{K}_{3 u}^{\sigma} \\
\mathbf{K}_{u 3}^{\sigma} & \overline{\mathbf{K}}_{u u}^{\sigma}
\end{array}\right]\left[\begin{array}{c}
v_{\mathrm{w}}^{3} \\
\mathbf{v}_{\mathrm{w}}^{u}
\end{array}\right] \psi^{\prime \prime \prime \prime}-\left[\begin{array}{cc}
K_{33}^{\tau} & \mathbf{K}_{3 u}^{\tau} \\
\mathbf{K}_{u 3}^{\tau} & \mathbf{K}_{u u}^{\tau}
\end{array}\right]\left[\begin{array}{c}
v_{\mathrm{w}}^{3} \\
\mathbf{v}_{\mathrm{w}}^{u}
\end{array}\right] \psi^{\prime \prime}} \\
+\left[\begin{array}{cc}
0 & \mathbf{0} \\
\mathbf{0} & \mathbf{K}_{u u}^{s}
\end{array}\right]\left[\begin{array}{c}
v_{\mathrm{w}}^{3} \\
\mathbf{v}_{\mathrm{w}}^{u}
\end{array}\right] \psi=\left[\begin{array}{c}
r_{\mathrm{w}}^{3} \\
\mathbf{r}_{\mathrm{w}}^{u \alpha}
\end{array}\right] \phi+\left[\begin{array}{c}
\bar{r}_{\Omega}^{3} \\
\overline{\mathbf{r}}_{\Omega}^{u \alpha}
\end{array}\right] \phi^{\prime}
\end{gathered}
$$

The stiffness matrix $\overline{\mathbf{K}}_{u u}^{\sigma}$ is found in the companion paper [1] and the vectors $\mathbf{r}_{\mathrm{w}}^{u \alpha}$ and $\overline{\mathbf{r}}_{\Omega}^{u \alpha}$ are given in Table 4. This equation constitutes the GBT differential equations constrained by shear flow constraints and wallwidth constraints after the elimination of the classical axial and two translational (flexural beam) modes.

\subsection{Step III - Reduction of order and torsional load}

The fourth order differential equation (43) can be transformed into twice as many second order differ- 
ential equations by introducing a so called state vector. There are a number of different possible formulations, however we choose the use of the state vector $\left(v_{\mathrm{w}}^{3} \psi, \mathbf{v}_{\mathrm{w}}^{u} \psi, v_{\mathrm{w}}^{3} \psi^{\prime \prime}, \mathbf{v}_{\mathrm{w}}^{u} \psi^{\prime \prime}\right)^{T}$. By introducing this state vector we obtain a reformulation of equation (43), leading to a formal second order matrix differential equation of double size, which takes the form

$$
\begin{gathered}
{\left[\begin{array}{cccc}
0 & \mathbf{0} & 0 & \mathbf{0} \\
\mathbf{0} & \mathbf{K}_{u u}^{s} & \mathbf{0} & \mathbf{0} \\
0 & \mathbf{0} & -K_{33}^{\sigma} & -\mathbf{K}_{3 u}^{\sigma} \\
\mathbf{0} & \mathbf{0} & -\mathbf{K}_{u 3}^{\sigma} & -\overline{\mathbf{K}}_{u u}^{\sigma}
\end{array}\right]\left[\begin{array}{c}
v_{\mathrm{w}}^{3} \psi \\
\mathbf{v}_{\mathrm{w}}^{u} \psi \\
v_{\mathrm{w}}^{3} \psi^{\prime \prime} \\
\mathbf{v}_{\mathrm{w}}^{u} \psi^{\prime \prime}
\end{array}\right]} \\
-\left[\begin{array}{cccc}
K_{33}^{\tau} & \mathbf{K}_{3 u}^{\tau} & -K_{33}^{\sigma} & -\mathbf{K}_{3 u}^{\sigma} \\
\mathbf{K}_{u 3}^{\tau} & \mathbf{K}_{u u}^{\tau} & -\mathbf{K}_{u 3}^{\sigma} & -\overline{\mathbf{K}}_{u u}^{\sigma} \\
-K_{33}^{\sigma} & -\mathbf{K}_{3 u}^{\sigma} & 0 & \mathbf{0} \\
-\mathbf{K}_{u 3}^{\sigma} & -\overline{\mathbf{K}}_{u u}^{\sigma} & \mathbf{0} & \mathbf{0}
\end{array}\right]\left[\begin{array}{c}
v_{\mathrm{w}}^{3} \psi \\
\mathbf{v}_{\mathrm{w}}^{u} \psi \\
v_{\mathrm{w}}^{3} \psi^{\prime \prime} \\
\mathbf{v}_{\mathrm{w}}^{u} \psi^{\prime \prime}
\end{array}\right] \\
=\left[\begin{array}{c}
r_{\mathrm{w}}^{3} \\
\mathbf{r}_{\mathrm{w}}^{u \alpha} \\
0 \\
\mathbf{0}
\end{array}\right] \phi+\left[\begin{array}{c}
\bar{r}_{\Omega}^{3} \\
\overline{\mathbf{r}}_{\Omega}^{u \alpha} \\
0 \\
\mathbf{0}
\end{array}\right] \phi^{\prime}
\end{gathered}
$$

To keep the matrix operations as simple as possible we introduce a new vector $\mathbf{v}_{\mathrm{w}}^{e}$, three new block matrices, $\mathbf{K}_{e e}^{\sigma}, \mathbf{K}_{3 e}^{\sigma}$, and $\mathbf{K}_{u e}^{\sigma}$ given by

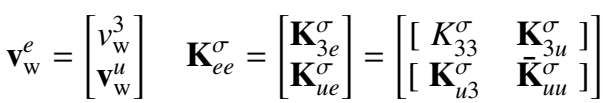

and the force vectors are given by

$\mathbf{r}_{\mathrm{w}}^{e}=\left[\begin{array}{l}0 \\ \mathbf{0}\end{array}\right] \quad, \quad \mathbf{r}_{\Omega}^{e}=\left[\begin{array}{l}0 \\ \mathbf{0}\end{array}\right]$

Introducing the new vectors and block matrices defined by equation (45) and (46), and the transformed loads given in Table 5 , the second order differential equations (44) can be rewritten as

$$
\begin{aligned}
& {\left[\begin{array}{ccc}
0 & \mathbf{0} & \mathbf{0} \\
\mathbf{0} & \mathbf{K}_{u u}^{s} & \mathbf{0} \\
\mathbf{0} & \mathbf{0} & -\mathbf{K}_{e e}^{\sigma}
\end{array}\right]\left[\begin{array}{c}
v_{\mathrm{w}}^{3} \psi \\
\mathbf{v}_{\mathrm{w}}^{u} \psi \\
\mathbf{v}_{\mathrm{w}}^{e} \psi^{\prime \prime}
\end{array}\right]} \\
& -\left[\begin{array}{ccc}
K_{33}^{\tau} & \mathbf{K}_{3 u}^{\tau} & -\mathbf{K}_{3 e}^{\sigma} \\
\mathbf{K}_{u 3}^{\tau} & \mathbf{K}_{u u}^{\tau} & -\mathbf{K}_{u e}^{\sigma} \\
-\mathbf{K}_{e 3}^{\sigma} & -\mathbf{K}_{e u}^{\sigma} & \mathbf{0}
\end{array}\right]\left[\begin{array}{c}
v_{\mathrm{w}}^{3} \psi \\
\mathbf{v}_{\mathrm{w}}^{u} \psi \\
\mathbf{v}_{\mathrm{w}}^{e} \psi^{\prime \prime}
\end{array}\right]^{\prime \prime}=\left[\begin{array}{c}
r_{\mathrm{w}}^{3} \\
\mathbf{r}_{\mathrm{w}}^{u \alpha} \\
\mathbf{r}_{\mathrm{w}}^{e}
\end{array}\right] \phi+\left[\begin{array}{c}
\bar{r}_{\Omega}^{3} \\
\overline{\mathbf{r}}_{\Omega}^{u \alpha} \\
\mathbf{r}_{\Omega}^{e}
\end{array}\right] \phi^{\prime}
\end{aligned}
$$

From the first equation we can isolate the pure rotational term resulting in the following differential equation:

$$
\begin{aligned}
& v_{\mathrm{w}}^{3} \psi^{\prime \prime}= \\
& \quad-\left(K_{33}^{\tau}\right)^{-1}\left(\mathbf{K}_{3 u}^{\tau} \mathbf{v}_{\mathrm{w}}^{u} \psi^{\prime \prime}-\mathbf{K}_{3 e}^{\sigma} \mathbf{v}_{\mathrm{w}}^{e} \psi^{\prime \prime \prime \prime}+r_{\mathrm{w}}^{3} \phi+\bar{r}_{\Omega}^{3} \phi^{\prime}\right)
\end{aligned}
$$

It can be seen that pure torsion (with free warping), corresponding to the solution vector, $\left(v_{\mathrm{w}}^{3} \psi, \mathbf{v}_{\mathrm{w}}^{u} \psi, \mathbf{v}_{\mathrm{w}}^{e} \psi^{\prime \prime}\right)=$ $\left(c_{32} z+c_{31}, \mathbf{0}, \mathbf{0}\right)$, is a solution of the homogeneous second order differential equations in (47). Hereby the particular solution for the axial variation of the pure torsion mode is determined by double integration of particular part, and the full solution is found by addition of the homogeneous solution. This results in

$\psi_{3}(z)=c_{31}+c_{32} z-\iint\left(K_{33}^{\tau}\right)^{-1}\left(\bar{r}_{\mathrm{w}}^{3} \phi+\bar{r}_{\Omega}^{3} \phi^{\prime}\right) d z d z$

Inserting the linear function $\phi$ from equation (31) we can evaluate the integrals in (49) and find the full solution of pure St. Venant torsion as

$\psi_{3}(z)=\boldsymbol{\Psi}_{3 h}(z) \mathbf{c}_{3}+\boldsymbol{\Psi}_{3 p}(z) \boldsymbol{\phi}$

Here $\mathbf{c}_{3}$ is a vector containing two constants of the homogeneous part of the solution, and

$$
\begin{aligned}
& \boldsymbol{\Psi}_{3 p}(z)=\boldsymbol{\Psi}_{3 p}^{\mathrm{w}}(z)+\boldsymbol{\Psi}_{3 p}^{\Omega}(z) \\
& \boldsymbol{\Psi}_{3 h}(z)=\left[\begin{array}{cc}
1 & z
\end{array}\right] \\
& \boldsymbol{\Psi}_{3 p}^{\mathrm{w}}(z)=-\frac{L^{2} r_{\mathrm{w}}^{3}}{6 K_{33}^{\tau}}\left[3\left(\frac{z}{L}\right)^{2}-\left(\frac{z}{L}\right)^{3} \quad\left(\frac{z}{L}\right)^{3}\right] \\
& \boldsymbol{\Psi}_{3 p}^{\Omega}(z)=-\frac{L \bar{r}_{\Omega}^{3}}{2 K_{33}^{\tau}}\left[\begin{array}{ll}
2\left(\frac{z}{L}\right)-\left(\frac{z}{L}\right)^{2} & \left(\frac{z}{L}\right)^{2}
\end{array}\right]
\end{aligned}
$$

Using equation (48) we eliminate $v_{\mathrm{w}}^{3}$ from the differential equations in (47) and find the final distortional nonhomogeneous differential equations of GBT that determine all the distortional displacement modes as

$$
\begin{array}{r}
{\left[\begin{array}{cc}
\mathbf{K}_{u u}^{s} & \mathbf{0} \\
\mathbf{0} & -\mathbf{K}_{e e}^{\sigma}
\end{array}\right]\left[\begin{array}{c}
\mathbf{v}_{\mathrm{w}}^{u} \psi \\
\mathbf{v}_{\mathrm{w}}^{e} \psi^{\prime \prime}
\end{array}\right]-\left[\begin{array}{cc}
\overline{\mathbf{K}}_{u u}^{\tau} & -\overline{\mathbf{K}}_{u e}^{\sigma} \\
-\overline{\mathbf{K}}_{e u}^{\sigma} & -\overline{\mathbf{K}}_{e e}^{\sigma}
\end{array}\right]\left[\begin{array}{c}
\mathbf{v}_{\mathrm{w}}^{u} \psi \\
\mathbf{v}_{\mathrm{w}}^{e} \psi^{\prime \prime}
\end{array}\right]^{\prime \prime}} \\
=\left[\begin{array}{c}
\mathbf{r}_{\mathrm{w}}^{\alpha u 3} \\
\mathbf{r}_{\mathrm{w}}^{e 3}
\end{array}\right] \phi+\left[\begin{array}{c}
\overline{\mathbf{r}}_{\Omega}^{\alpha u 3} \\
\mathbf{r}_{\Omega}^{e 3}
\end{array}\right] \phi^{\prime}
\end{array}
$$

The block matrices and the transformed stiffness matrices are introduced in the companion paper [1] and the load vectors are given in Table 5.

$$
\begin{array}{ll}
\mathbf{r}_{\mathrm{w}}^{\alpha u 3}=\mathbf{r}_{\mathrm{w}}^{u \alpha}-\mathbf{K}_{u 3}^{\tau} K_{33}^{\tau}{ }^{-1} r_{\mathrm{w}}^{3} & \mathbf{r}_{\mathrm{w}}^{e 3}=\mathbf{r}_{\mathrm{w}}^{e}+\mathbf{K}_{e 3}^{\sigma} K_{33}^{\tau}{ }^{-1} r_{\mathrm{w}}^{3} \\
\overline{\mathbf{r}}_{\Omega}^{\alpha u 3}=\overline{\mathbf{r}}_{\Omega}^{u \alpha}-\mathbf{K}_{u 3}^{\tau} K_{33}^{\tau}{ }^{-1} \bar{r}_{\Omega}^{3} & \mathbf{r}_{\Omega}^{e 3}=\mathbf{r}_{\Omega}^{e}+\mathbf{K}_{e 3}^{\sigma} K_{33}^{\tau}{ }^{-1} \bar{r}_{\Omega}^{3} \\
\hline
\end{array}
$$

Table 5: Transformation of load vectors related to Step III.

\section{Solution of distortional equations}

The distortional eigenvalue problem for the homogeneous system of equations (55) was solved in the companion paper [1]. Here the eigenvalues, $\lambda_{i}=\xi_{i}^{2}$, and the 
corresponding eigenvectors was given by

$\left[\begin{array}{c}\mathbf{v}_{\mathrm{w}}^{u} \\ \mathbf{v}_{\mathrm{w}}^{e} \xi^{2}\end{array}\right]_{i}=\left[\begin{array}{c}\mathbf{v}_{\mathrm{w} i}^{u} \\ \mathbf{v}_{\mathrm{w} i}^{e} \xi_{i}^{2}\end{array}\right]$

In the presents context these eigenvectors can be used to decouple the system of equations in (55). The $i$ 'th decoupled equation which determines the axial variation $\psi_{d i}(z)$ of the distortional eigenvector is found by inserting the $i$ 'th eigenvector and pre multiplying by it, which results in the following equation:

$$
\begin{gathered}
{\left[\begin{array}{c}
\mathbf{v}_{\mathrm{w}}^{u} \\
\mathbf{v}_{\mathrm{w}}^{e} \xi^{2}
\end{array}\right]_{i}^{T}\left[\begin{array}{cc}
\mathbf{K}_{u u}^{s} & \mathbf{0} \\
\mathbf{0} & -\mathbf{K}_{e e}^{\sigma}
\end{array}\right]\left[\begin{array}{c}
\mathbf{v}_{\mathrm{w}}^{u} \\
\mathbf{v}_{\mathrm{w}}^{e} \xi^{2}
\end{array}\right]_{i} \psi_{d i}} \\
-\left[\begin{array}{c}
\mathbf{v}_{\mathrm{w}}^{u} \\
\mathbf{v}_{\mathrm{w}}^{e} \xi^{2}
\end{array}\right]_{i}^{T}\left[\begin{array}{cc}
\overline{\mathbf{K}}_{u u}^{\tau} & -\overline{\mathbf{K}}_{u e}^{\sigma} \\
-\overline{\mathbf{K}}_{e u}^{\sigma} & -\overline{\mathbf{K}}_{e e}^{\sigma}
\end{array}\right]\left[\begin{array}{c}
\mathbf{v}_{\mathrm{w}}^{u} \\
\mathbf{v}_{\mathrm{w}}^{e} \xi^{2}
\end{array}\right]_{i} \psi_{d i}^{\prime \prime} \\
=\left[\begin{array}{c}
\mathbf{v}_{\mathrm{w}}^{u} \\
\mathbf{v}_{\mathrm{w}}^{e} \xi^{2}
\end{array}\right]_{i}^{T}\left[\begin{array}{c}
\mathbf{r}_{\mathrm{w}}^{\alpha u 3} \\
\mathbf{r}_{\mathrm{w}}^{e 3}
\end{array}\right] \phi+\left[\begin{array}{c}
\mathbf{v}_{\mathrm{w}}^{u} \\
\mathbf{v}_{\mathrm{w}}^{e} \xi^{2}
\end{array}\right]_{i}^{T}\left[\begin{array}{c}
\overline{\mathbf{r}}_{\Omega}^{\alpha u 3} \\
\mathbf{r}_{\Omega}^{e 3}
\end{array}\right] \phi^{\prime}
\end{gathered}
$$

which we abbreviate as

$K_{i i}^{g} \psi_{d_{i}}-K_{i i}^{d} \psi_{d_{i}}^{\prime \prime}=r_{\mathrm{w} i}^{d} \phi+r_{\Omega i}^{d} \phi^{\prime}$

Normalizing this equation and introducing that the eigenvalue $\xi_{i}{ }^{2}$ is equal to $K_{i i}^{g} / K_{i i}^{d}$, it takes the following standard form

$\psi_{d_{i}}^{\prime \prime}-\xi_{i}^{2} \psi_{d_{i}}=-\frac{1}{K_{i i}^{d}}\left(r_{\mathrm{w} i}^{d} \phi+r_{\Omega i}^{d} \phi^{\prime}\right)$

The above introduced distortional stiffness and load terms are given in Table 6 . Note that $r_{\mathrm{w} i}^{d} \psi$ is the distortional moment load and $r_{\Omega i}^{d} \psi^{\prime}$ is the distortional bimoment load.

We find that the full solution of each of these uncoupled non-homogeneous linear 2 . order differential equations is given by

$$
\begin{aligned}
\psi_{d i}(z)= & c_{1} e^{\xi_{i} z}+c_{2} e^{-\xi_{i} z} \\
& -\frac{1}{2 \xi_{i}} e^{\xi_{i} z} \int e^{-\xi_{i} z} \frac{1}{K_{i i}^{d}}\left(r_{\mathrm{w} i}^{d} \phi+r_{\Omega i}^{d} \phi^{\prime}\right) d z \\
& +\frac{1}{2 \xi_{i}} e^{-\xi_{i} z} \int e^{\xi_{i} z} \frac{1}{K_{i i}^{d}}\left(r_{\mathrm{w} i}^{d} \phi+r_{\Omega i}^{d} \phi^{\prime}\right) d z
\end{aligned}
$$

Using that $\phi$ is a linear function as given in equation (31) and performing integration or by guessing the solution we get

$\psi_{d_{i}}(z)=\boldsymbol{\Psi}_{d h_{i}}(z) \mathbf{c}_{d_{i}}+\boldsymbol{\Psi}_{d p_{i}}(z) \boldsymbol{\phi}$

Here $\mathbf{c}_{d i}$ is a vector containing the two constants $c_{d_{2 i-1}}$ and $c_{d_{2 i}}$ of the homogeneous part of the solution, and

$$
\begin{aligned}
& \boldsymbol{\Psi}_{d p_{i}}(z)=\boldsymbol{\Psi}_{d p_{i}}^{\mathrm{w}}(z)+\boldsymbol{\Psi}_{d p_{i}}^{\Omega}(z) \\
& \boldsymbol{\Psi}_{d h_{i}}(z)=\left[\begin{array}{ll}
e^{\xi_{i} z} & e^{-\xi_{i} z}
\end{array}\right] \\
& \boldsymbol{\Psi}_{d p_{i}}^{\mathrm{w}}=\frac{r_{\mathrm{w} i}^{d}}{\xi_{i}{ }^{2} K_{i i}^{d}}\left[\begin{array}{ll}
1-\frac{z}{L} & \frac{z}{L}
\end{array}\right] \\
& \boldsymbol{\Psi}_{d p_{i}}^{\Omega}=\frac{r_{\Omega i}^{d}}{\xi_{i}{ }^{2} K_{i i}^{d}}\left[\begin{array}{ll}
\frac{-1}{L} & \frac{1}{L}
\end{array}\right]
\end{aligned}
$$

This concludes the determination of all the solutions for all the displacement modes of GBT.

\section{Assembly of the full solution}

The axial variation of the four beam modes have been identified in equations (32), (37) and (50) and can be assembled in the beam solution function matrices $\boldsymbol{\Psi}_{b h}(z)$ and $\boldsymbol{\Psi}_{b p}(z)$ which are multiplied by the vector of beam displacement constants $\mathbf{c}_{b}$ and the load vector $\boldsymbol{\phi}$ respectively. This results in

$$
\begin{aligned}
& \boldsymbol{\Psi}_{b h}(z) \mathbf{c}_{b}+\boldsymbol{\Psi}_{b p}(z) \boldsymbol{\phi}= \\
& {\left[\begin{array}{ccc}
\boldsymbol{\Psi}_{a h}(z) & \mathbf{0} & \mathbf{0} \\
\mathbf{0} & \boldsymbol{\Psi}_{\alpha h}(z) & \mathbf{0} \\
\mathbf{0} & \mathbf{0} & \boldsymbol{\Psi}_{3 h}(z)
\end{array}\right]\left[\begin{array}{l}
\mathbf{c}_{a} \\
\mathbf{c}_{\alpha} \\
\mathbf{c}_{3}
\end{array}\right]+\left[\begin{array}{l}
\boldsymbol{\Psi}_{a p}(z) \\
\boldsymbol{\Psi}_{\alpha p}(z) \\
\boldsymbol{\Psi}_{3 p}(z)
\end{array}\right] \boldsymbol{\phi}}
\end{aligned}
$$

where

$$
\begin{aligned}
& \boldsymbol{\Psi}_{\alpha h}(z)=\left[\begin{array}{cc}
\boldsymbol{\Psi}_{1 h}(z) & \mathbf{0} \\
\mathbf{0} & \boldsymbol{\Psi}_{2 h}(z)
\end{array}\right] \\
& \boldsymbol{\Psi}_{\alpha p}(z)=\left[\begin{array}{l}
\boldsymbol{\Psi}_{1 p}(z) \\
\boldsymbol{\Psi}_{2 p}(z)
\end{array}\right]
\end{aligned}
$$

Furthermore the distortional solution functions can be assembled and described as

$$
\begin{aligned}
& \boldsymbol{\Psi}_{d h}(z) \mathbf{c}_{d}+\boldsymbol{\Psi}_{d p}(z) \boldsymbol{\phi}= \\
& {\left[\begin{array}{cccc}
\boldsymbol{\Psi}_{d h_{1}}(z) & \mathbf{0} & \mathbf{0} & \cdots \\
\mathbf{0} & \boldsymbol{\Psi}_{d h_{2}}(z) & \mathbf{0} & \cdots \\
\mathbf{0} & \mathbf{0} & \boldsymbol{\Psi}_{d h_{3}}(z) & \cdots \\
\vdots & \vdots & \vdots & \ddots
\end{array}\right]\left[\begin{array}{c}
\mathbf{c}_{d_{1}} \\
\mathbf{c}_{d_{2}} \\
\mathbf{c}_{d_{3}} \\
\vdots
\end{array}\right]+\left[\begin{array}{c}
\boldsymbol{\Psi}_{d p_{1}}(z) \\
\boldsymbol{\Psi}_{d p_{2}}(z) \\
\boldsymbol{\Psi}_{d p_{3}}(z) \\
\vdots
\end{array}\right] \boldsymbol{\phi}}
\end{aligned}
$$

Now all the solution functions are obtained and can be assembled using the previously defined block matrices and vectors as

$\boldsymbol{\Psi}_{h}(z) \mathbf{c}+\boldsymbol{\Psi}_{p}(z) \boldsymbol{\phi}$

in which

$\boldsymbol{\Psi}_{\mathbf{h}}(z) \mathbf{c}=\left[\begin{array}{cc}\boldsymbol{\Psi}_{b h}(z) & \mathbf{0} \\ \mathbf{0} & \boldsymbol{\Psi}_{d h}(z)\end{array}\right]\left[\begin{array}{l}\mathbf{c}_{b} \\ \mathbf{c}_{d}\end{array}\right]$ 


$$
\begin{array}{rlr}
K_{i i}^{d}=\left[\begin{array}{c}
\mathbf{v}_{\mathrm{w}}^{u} \\
\mathbf{v}_{\mathrm{w}}^{e} \xi^{2}
\end{array}\right]_{i}^{T}\left[\begin{array}{cc}
\overline{\mathbf{K}}_{u u}^{\tau} & -\overline{\mathbf{K}}_{u e}^{\sigma} \\
-\overline{\mathbf{K}}_{e u}^{\sigma} & -\overline{\mathbf{K}}_{e e}^{\sigma}
\end{array}\right]\left[\begin{array}{c}
\mathbf{v}_{\mathrm{w}}^{u} \\
\mathbf{v}_{\mathrm{w}}^{e} \xi^{2}
\end{array}\right]_{i} & r_{\mathrm{w} i}^{d}=\left[\begin{array}{c}
\mathbf{v}_{\mathrm{w}}^{u} \\
\mathbf{v}_{\mathrm{w}}^{e} \xi^{2}
\end{array}\right]_{i}^{T}\left[\begin{array}{c}
\mathbf{r}_{\mathrm{w}}^{\alpha u 3} \\
\mathbf{r}_{\mathrm{w}}^{e 3}
\end{array}\right] \\
K_{i i}^{g}=\xi_{i}^{2} K_{i i}^{d}=\left[\begin{array}{c}
\mathbf{v}_{\mathrm{w}}^{u} \\
\mathbf{v}_{\mathrm{w}}^{e} \xi^{2}
\end{array}\right]_{i}^{T}\left[\begin{array}{cc}
\mathbf{K}_{u u}^{s} & \mathbf{0} \\
\mathbf{0} & -\mathbf{K}_{e e}^{\sigma}
\end{array}\right]\left[\begin{array}{c}
\mathbf{v}_{\mathrm{w}}^{u} \\
\mathbf{v}_{\mathrm{w}}^{e} \xi^{2}
\end{array}\right]_{i} & r_{\Omega i}^{d}=\left[\begin{array}{c}
\mathbf{v}_{\mathrm{w}}^{u} \\
\mathbf{v}_{\mathrm{w}}^{e} \xi^{2}
\end{array}\right]_{i}^{T}\left[\begin{array}{c}
\overline{\mathbf{r}}_{\Omega}^{\alpha u 3} \\
\mathbf{r}_{\Omega}^{e 3}
\end{array}\right]
\end{array}
$$

Table 6: Modal distortional stiffness and load terms.

and

$$
\boldsymbol{\Psi}_{\mathbf{p}}(z) \boldsymbol{\phi}=\left[\begin{array}{c}
\boldsymbol{\Psi}_{b p}(z) \\
\boldsymbol{\Psi}_{d p}(z)
\end{array}\right] \boldsymbol{\phi}
$$

As we are using the in-plane modes found in the companion paper [1], the back substitution process of distortional and eliminated beam displacement in-plane modes is identical to the process performed in the companion paper. Hereby all the in-plane modes are assembled column-wise in a modal matrix of transverse displacement vectors $\mathbf{V}_{\mathrm{w}}$ and a modal matrix of axial warping displacement vectors $\mathbf{V}_{\Omega}$, by joining the modal matrices of the beam eigenvectors and the distortional eigenvectors as

$\mathbf{V}_{\mathrm{w}}=\left[\begin{array}{ll}\mathbf{V}_{\mathrm{w}}^{b} & \mathbf{V}_{\mathrm{w}}^{d}\end{array}\right] \quad \mathbf{V}_{\Omega}=\left[\begin{array}{ll}\mathbf{V}_{\Omega}^{b} & \mathbf{V}_{\Omega}^{d}\end{array}\right]$

Having obtained and assembled all the full solution functions and in-plane modes, the full solution along the beam can be presented in the nodal solution vectors $\mathbf{u}_{\mathrm{w}}(z)$ and $\mathbf{u}_{\Omega}(z)$ as follows:

$$
\begin{aligned}
\mathbf{u}_{\mathrm{w}}(z) & =\mathbf{V}_{\mathrm{w}}\left[\boldsymbol{\Psi}_{h}(z) \mathbf{c}+\boldsymbol{\Psi}_{p}(z) \boldsymbol{\phi}\right] \\
\mathbf{u}_{z}(z) & =-\mathbf{V}_{\Omega}\left[\boldsymbol{\Psi}_{h}^{\prime}(z) \mathbf{c}+\boldsymbol{\Psi}_{p}^{\prime}(z) \boldsymbol{\phi}\right]
\end{aligned}
$$

The constants, $\mathbf{c}$, have to be determined by the boundary conditions of the thin-walled beam.

\subsection{Transformation to real modes and real solution functions}

Some of the distortional solution functions found are complex. Because these complex numbers are awkward to handle it is a matter of considerable importance to construct a more convenient complete solution when we have complex numbers. In [1] we introduce the following notation for the positive square root values and the related eigenvector columns $\mathbf{v}_{j}$ and $\mathbf{v}_{j+1}$ :

$$
\begin{array}{ll}
\xi_{j}=\lambda_{j}+\mu_{j} i & \xi_{j+1}=\bar{\xi}_{j}=\lambda_{j}-\mu_{j} i \\
\mathbf{v}_{j}=\mathbf{a}_{j}+\mathbf{b}_{j} i & \mathbf{v}_{j+1}=\overline{\mathbf{v}}_{j}=\mathbf{a}_{j}-\mathbf{b}_{j} i
\end{array}
$$

in which we have introduced the real and imaginary parts of the eigenvalues and eigenvectors. The complex eigenvectors in equation (76) may be conveniently written as

$$
\left[\begin{array}{ll}
\mathbf{v}_{j} & \mathbf{v}_{j+1}
\end{array}\right]=\left[\begin{array}{ll}
\mathbf{a}_{j} & \mathbf{b}_{j}
\end{array}\right]\left[\begin{array}{cc}
1 & 1 \\
i & -i
\end{array}\right]
$$

The constants of the related parts of the homogeneous solution are also complex quantities. However we are able to assemble the two complex conjugated modal solutions into two real (but pairwise coupled) solutions by introducing the real constant vectors $\widetilde{\mathbf{c}}_{j}$ and $\widetilde{\mathbf{c}}_{j+1}$ as follows:

$\left[\begin{array}{c}\mathbf{c}_{j} \\ \mathbf{c}_{j+1}\end{array}\right]=\frac{1}{2}\left[\begin{array}{cc}1 & -i \\ 1 & i\end{array}\right]\left[\begin{array}{c}\widetilde{\mathbf{c}}_{j} \\ \widetilde{\mathbf{c}}_{j+1}\end{array}\right]$

The $j$ 'th complex part of the full solution in equation (74) can now be rewritten using the transformations in equations (77) and (78). After multiplication and identification of real and imaginary parts we find the following result:

$$
\begin{array}{r}
{\left[\begin{array}{ll}
\mathbf{v}_{j} & \mathbf{v}_{j+1}
\end{array}\right]\left\{\left[\begin{array}{cc}
\boldsymbol{\Psi}_{d h_{j}} & \mathbf{0} \\
\mathbf{0} & \overline{\boldsymbol{\Psi}}_{d h_{j}}
\end{array}\right]\left[\begin{array}{c}
\mathbf{c}_{j} \\
\mathbf{c}_{j+1}
\end{array}\right]+\left[\begin{array}{l}
\boldsymbol{\Psi}_{d p_{j}} \\
\overline{\boldsymbol{\Psi}}_{d p_{j}}
\end{array}\right]\left[\begin{array}{l}
\phi_{1} \\
\phi_{2}
\end{array}\right]\right\}} \\
=\left[\begin{array}{ll}
\mathbf{a}_{j} & \mathbf{b}_{j}
\end{array}\right]\left\{\left[\begin{array}{cc}
\operatorname{Re}\left(\boldsymbol{\Psi}_{d h_{j}}\right) & \operatorname{Im}\left(\boldsymbol{\Psi}_{d h_{j}}\right) \\
\operatorname{Im}\left(\overline{\boldsymbol{\Psi}}_{d h_{j}}\right) & \operatorname{Re}\left(\overline{\boldsymbol{\Psi}}_{d h_{j}}\right)
\end{array}\right]\left[\begin{array}{c}
\widetilde{\mathbf{c}}_{j} \\
\widetilde{\mathbf{c}}_{j+1}
\end{array}\right]\right. \\
\left.+\left[\begin{array}{c}
2 \operatorname{Re}\left(\boldsymbol{\Psi}_{d p_{j}}\right) \\
-2 \operatorname{Im}\left(\overline{\boldsymbol{\Psi}}_{d p_{j}}\right)
\end{array}\right]\left[\begin{array}{l}
\phi_{1} \\
\phi_{2}
\end{array}\right]\right\}
\end{array}
$$

Hereby it is possible to rewrite the complex quantities into real quantities. Now the modal matrices $\mathbf{V}_{\mathrm{w}}$ and $\mathbf{V}_{\Omega}$ are modified as $\widetilde{\mathbf{V}}_{\mathrm{w}}$ and $\widetilde{\mathbf{V}}_{\Omega}$ by substituting the complex pairs of eigenvectors with their respective real and imaginary parts. Furthermore we also introduce the modified solution matrices $\widetilde{\boldsymbol{\Psi}}_{d h}$ and $\widetilde{\boldsymbol{\Psi}}_{d p}$ and the related modified vector of constants $\widetilde{\mathbf{c}}$ by substituting the solutions (and constants) of the complex pairs using equation (79). Now the full solution along the beam can be written using real numbers as

$$
\begin{aligned}
& \mathbf{u}_{\mathrm{w}}(z)=\widetilde{\mathbf{V}}_{\mathrm{w}}\left[\widetilde{\boldsymbol{\Psi}}_{h}(z) \widetilde{\mathbf{c}}+\widetilde{\boldsymbol{\Psi}}_{p}(z) \boldsymbol{\phi}\right] \\
& \mathbf{u}_{z}(z)=-\widetilde{\mathbf{V}}_{\Omega}\left[\widetilde{\boldsymbol{\Psi}}_{h}^{\prime}(z) \widetilde{\mathbf{c}}+\widetilde{\boldsymbol{\Psi}}_{p}^{\prime}(z) \boldsymbol{\phi}\right]
\end{aligned}
$$


Hereby it is possible to work with this real formulation or continue working with complex numbers using the full solution formulated in equation (74). In the following context we will use the real formulation in equation (80).

\section{Displacement boundary conditions}

In this section we will introduce a method for determining the constants of the non-homogeneous solutions found. This is to be done in the GBT space, which has been constrained by the relevant assumptions of the beam theory. As seen from the first variation of the potential energy, the natural boundary displacements of the GBT at each boundary are the pure axial displacement $u_{\Omega}^{a}$ of the beam, the transverse displacements $\mathbf{u}_{\mathrm{w}}^{g}$, and the axial derivative of the transverse displacements $\mathbf{u}_{\mathrm{w}}^{g}{ }^{\prime}$. In the following the generalized internal displacements of the GBT beam will be expressed by using the transformation from FE to GBT displacements as follows:

$\left[\begin{array}{c}u_{z}(z) \\ \mathbf{u}_{\mathrm{w}}^{g}(z) \\ \mathbf{u}_{\mathrm{w}}^{g \prime}(z)\end{array}\right]=\left[\begin{array}{c}-\mathbf{T}_{\Omega}^{a T} \widetilde{\mathbf{V}}_{\Omega} \widetilde{\boldsymbol{\Psi}}_{h}^{\prime}(z) \\ \mathbf{T}_{\mathrm{w}}^{g} T \widetilde{\mathbf{V}}_{\mathrm{w}} \widetilde{\boldsymbol{\Psi}}_{h}(z) \\ \mathbf{T}_{\mathrm{w}}^{g T} \widetilde{\mathbf{V}}_{\mathrm{w}} \widetilde{\boldsymbol{\Psi}}_{h}^{\prime}(z)\end{array}\right] \widetilde{\mathbf{c}}+\left[\begin{array}{c}-\mathbf{T}_{\Omega}^{a T} \widetilde{\mathbf{V}}_{\Omega} \widetilde{\boldsymbol{\Psi}}_{p}^{\prime}(z) \\ \mathbf{T}_{\mathrm{w}}^{g T} \widetilde{\mathbf{V}}_{\mathrm{w}} \widetilde{\boldsymbol{\Psi}}_{p}(z) \\ \mathbf{T}_{\mathrm{w}}^{g} T \widetilde{\mathbf{V}}_{\mathrm{w}} \widetilde{\boldsymbol{\Psi}}_{p}^{\prime}(z)\end{array}\right] \boldsymbol{\phi}(81)$

To determine the constants using displacement boundary conditions as in finite element or stiffness formulations, the boundary displacements at the two ends of a finite length beam are needed, i.e. at $z=0$ and at $z=L$, where $L$ is the length of the beam. The assembled boundary displacement vector is denoted by $\mathbf{u}_{b}$. This leads to the following equation for the determination of the solution constants:

$$
\begin{aligned}
& \mathbf{u}_{b}=\left[\begin{array}{c}
u_{z}^{a}(0) \\
\mathbf{u}_{\mathrm{w}}^{g}(0) \\
\mathbf{u}_{\mathrm{w}}^{g}(0) \\
u_{z}^{a}(L) \\
\mathbf{u}_{\mathrm{w}}^{g}(L) \\
\mathbf{u}_{\mathrm{w}}^{g \prime}(L)
\end{array}\right] \\
& =\left[\begin{array}{c}
-\mathbf{T}_{\Omega}^{a T} \widetilde{\mathbf{V}}_{\Omega} \widetilde{\boldsymbol{\Psi}}_{h}^{\prime}(0) \\
\mathbf{T}_{\mathrm{w}}^{g T} \widetilde{\mathbf{V}}_{\mathrm{w}} \widetilde{\boldsymbol{\Psi}}_{h}(0) \\
\mathbf{T}_{\mathrm{w}}^{g T} \widetilde{\mathbf{V}}_{\mathrm{w}} \widetilde{\boldsymbol{\Psi}}_{h}^{\prime}(0) \\
-\mathbf{T}_{\Omega}^{a T} \widetilde{\mathbf{V}}_{\Omega} \widetilde{\boldsymbol{\Psi}}_{h}^{\prime}(L) \\
\mathbf{T}_{\mathrm{w}}^{g T} \widetilde{\mathbf{V}}_{\mathrm{w}} \widetilde{\boldsymbol{\Psi}}_{h}(L) \\
\mathbf{T}_{\mathrm{w}}^{g T} \widetilde{\mathbf{V}}_{\mathrm{w}} \widetilde{\boldsymbol{\Psi}}_{h}^{\prime}(L)
\end{array}\right] \widetilde{\mathbf{c}}+\left[\begin{array}{r}
-\mathbf{T}_{\Omega}^{a T} \widetilde{\mathbf{V}}_{\Omega} \widetilde{\boldsymbol{\Psi}}_{p}^{\prime}(0) \\
\mathbf{T}_{\mathrm{w}}^{g T} \widetilde{\mathbf{V}}_{\mathrm{w}} \widetilde{\mathbf{\Psi}}_{p}(0) \\
\mathbf{T}_{\mathrm{w} T}^{g T} \widetilde{\mathbf{V}}_{\mathrm{w}} \widetilde{\boldsymbol{\Psi}}_{p}^{\prime}(0) \\
-\mathbf{T}_{\Omega}^{a T} \widetilde{\mathbf{V}}_{\Omega} \widetilde{\boldsymbol{\Psi}}_{p}^{\prime}(L) \\
\mathbf{T}_{\mathrm{w}}^{g T} \widetilde{\mathbf{V}}_{\mathrm{w}} \widetilde{\boldsymbol{\Psi}}_{p}(L) \\
\mathbf{T}_{\mathrm{w}}^{g T} \widetilde{\mathbf{V}}_{\mathrm{w}} \widetilde{\boldsymbol{\Psi}}_{p}^{\prime}(L)
\end{array}\right] \boldsymbol{\phi} \\
& =\widetilde{\mathbf{A}} \widetilde{\mathbf{c}}+\widetilde{\mathbf{B}} \boldsymbol{\phi} \\
& \Rightarrow \quad \widetilde{\mathbf{c}}=\widetilde{\mathbf{A}}^{-1}\left(\mathbf{u}_{b}-\widetilde{\mathbf{B}} \boldsymbol{\phi}\right)
\end{aligned}
$$

where we have introduced the matrix $\widetilde{\mathbf{A}}$ and $\widetilde{\mathbf{B}}$, were $\widetilde{\mathbf{A}}$ is an invertible positive definite "square" matrix. To avoid numerical problems the exponential solution functions in $\widetilde{\boldsymbol{\Psi}}_{h}(z)$ may have to be modified by replacing $\widetilde{c}_{i} e^{\xi_{i} z}$ by $\hat{c}_{i} e^{\xi_{i}(z-L)}$ so that the positive $\xi_{i} z$ exponent is bounded.

To see the effect of the load as it would be in a finite element context with built in edges, we choose to plot the solution by using equation (80), with all boundary displacements being zero (built in) as

$\mathbf{u}_{b}^{T}=\left[\begin{array}{llllll}0 & \mathbf{0} & \mathbf{0} & 0 & \mathbf{0} & \mathbf{0}\end{array}\right]^{T}$

This is done in the following examples.

\section{Examples}

In this section four examples will be given and nodal displacement results as well as stress distribution results of GBT will be compared to those found using the commercial FE program Abaqus. In the examples we consider a lipped channel and a box beam, both for two different load cases. For all load cases the loads are uniformly distributed and thus given by a cross-section load distribution multiplied by $\phi(z)=1$. In all four examples the beams have a length of $L=2000 \mathrm{~mm}$, an elasticity modulus $E=2.1 \cdot 10^{5} \mathrm{MPa}$ and a Poisson ratio of $v=0.3$.

The results found using Abaqus are based on isotropic material and the S4 shell element with full 4 point integration. The linear elastic finite element calculations are based on a structured rectangular mesh with a side length seed of $5 \mathrm{~mm}$.

\subsection{Example 1-Flexural load on lipped channel}

Using the full solution in equation (80) with parameters, discretization and distributed cross-section load as given and shown in Figure 5 leads to the deformations shown in Figure 6. Here it is seen that the main deformation is related to flexure, however also an in-plane deformation of the cross section becomes clear. This points out the importance of taking distortion into account in order to obtain a good approximation of the deformation shape even in simple load cases.

Comparing a nodal displacement of GBT to the one found from a model in the commercial FE program Abaqus, gives the values and the corresponding deviations shown in Table 7 . The values corresponds to the node marked on the deformed plot of the GBT solution in Figure 6 at mid-span of the beam. From Table 7 the deviation from Abaqus results of the displacement, $u_{x}$, in the horizontal direction is $0.6 \%$. As the present approach is based on a beam theory this deviation may 


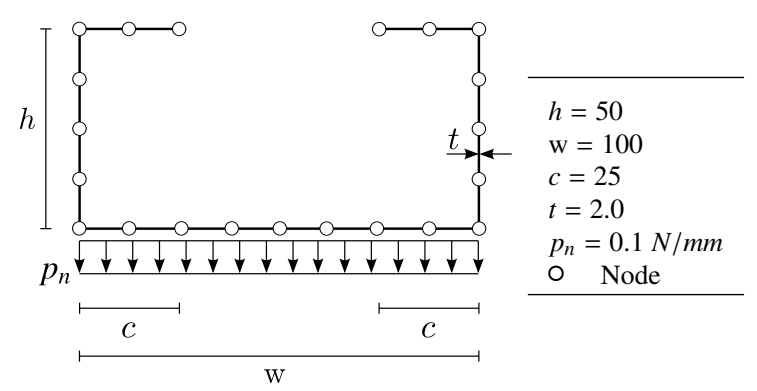

Figure 5: Geometry, parameter values and load for the lipped channel.

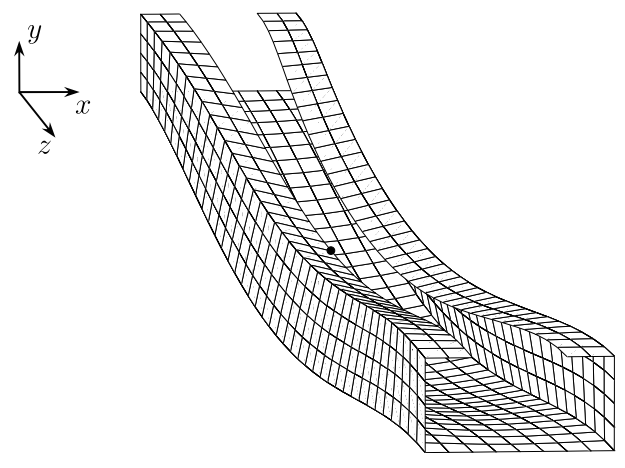

Figure 6: GBT plot of the lipped channel with a flexural load.

be expected. In contrast, the deviation for the vertical displacement, $u_{y}$, is as large as $3.3 \%$, however this can be explained by the formulation of the present theory, which is based on a beam theory where the shear deformations are neglected. In our Abaqus model we are using shell elements which include in-plane shear deformation. Calculating the contribution of shear deformations to the displacement as follows gives

$\Delta u_{y}(z)=\frac{1}{8} \frac{p L^{2}}{A_{w e b} G}=0.31 \mathrm{~mm}$

which is based on a web area of $A_{\text {web }}=2 h t$ and a load of $p=p_{n} \mathrm{w}$ in which $h$ is the height of one of the two webs and $\mathrm{w}$ is the width of the loaded flange. Adding this contribution to the vertical GBT displacement value in Table 7 gives $u_{y}=10.844 \mathrm{~mm}+0.3 \mathrm{~mm}=11.144 \mathrm{~mm}$, which then corresponds to a deviation of $0.6 \%$. Having

\begin{tabular}{rrrr} 
& GBT [mm] & Abaqus [mm] & Difference [\%] \\
\hline$u_{x}$ & 2.409 & 2.395 & 0.6 \\
$u_{y}$ & -10.844 & -11.213 & 3.3 \\
\hline
\end{tabular}

Table 7: Example 1: Nodal displacements of GBT and FE analysis.

compared the nodal displacement obtained with GBT and Abaqus we will now take a look at the stress distributions. A comparison of the membrane stresses in the $z$ direction at mid-span are shown in Figure 7 which gives a maximum deviation of $0.5 \%$ as shown in Table 8. As the present approach is based on a beam theory



Figure 7: Comparison between the axial normal stress distributions obtained with GBT and Abaqus at mid-span. All values are in MPa.

\begin{tabular}{rrrr} 
& GBT [MPa] & Abaqus [MPa] & Difference [\%] \\
\hline$\sigma_{z}$ & 154.0 & 154.8 & 0.5 \\
$\sigma_{s}$ & -73.1 & -69.4 & 5.3 \\
\hline
\end{tabular}

Table 8: Example 1: Stress distributions of GBT and FE analysis.



Figure 8: Comparison between the transverse bending stress distributions obtained with GBT and Abaqus at mid-span. All values are in MPa.

this deviation may be expected. The transverse bending stresses at mid-span are shown in Figure 8 and shows a maximum deviation in the corner at the bottom of $5.3 \%$ which is caused by the approach based on a beam theory and by the chosen mesh size. Reducing the mesh side length seed to $3 \mathrm{~mm}$ the maximum deviation is reduced to $3.1 \%$.

\subsection{Example 2 - Distortional load on lipped channel}

In this example the same lipped channel cross section as in the first example is now loaded by a distortional load as shown in Figure 9. Solving the equations leads to the GBT deformation solution shown in Figure 10 , which has displacements of the lips in both transverse coordinate directions with the maximum value at mid-span. It is seen that the distortional deformation dominates and that the boundary conditions give raise to relatively local end effects, whereas the deformations around mid span are relatively constant. Comparing the nodal displacements of the node marked in Figure 10 


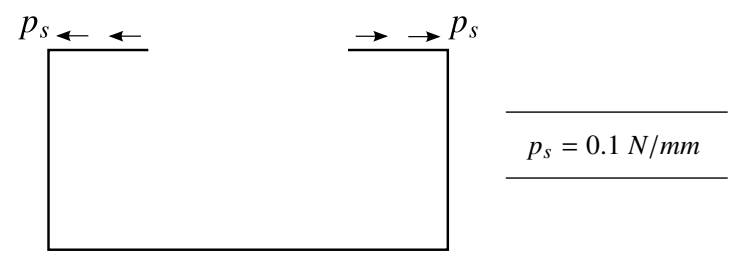

Figure 9: Distributed distortional load.

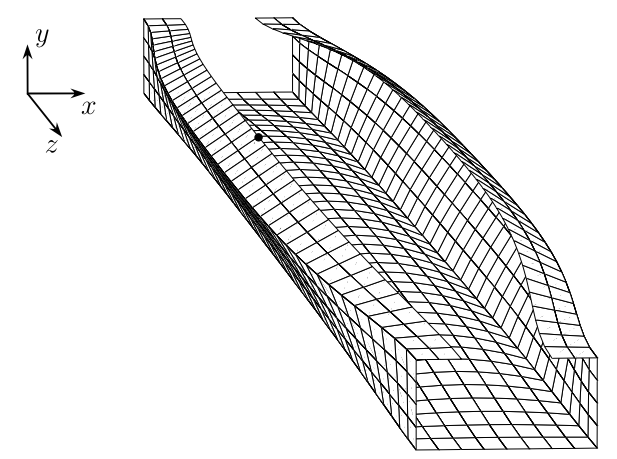

Figure 10: GBT plot of the lipped channel with a distortional load.

to the displacements found using a model in the commercial FE program Abaqus gives the displacement values and the corresponding deviations shown in Table 9. Here the deviation according to the maximum displacement in the horizontal direction, $u_{x}$, is $0.2 \%$ and the deviation for the vertical direction, $u_{y}$, is $0.4 \%$. Again we

\begin{tabular}{rrrr} 
& GBT [mm] & Abaqus [mm] & Difference [\%] \\
\hline$u_{x}$ & -2.847 & -2.841 & 0.2 \\
$u_{y}$ & 2.093 & 2.084 & 0.4 \\
\hline
\end{tabular}

Table 9: Example 2: Nodal displacements of GBT and FE analysis.

also want a comparison between the stress distributions obtained with GBT and Abaqus. In order to have comparable values different from zero the results concerning the axial normal stresses are obtained from the end section. A comparison of the axial membrane stresses in the $z$ direction are shown in Figure 11 and shows a maximum deviation of $7.9 \%$ as given in Table 10 . This
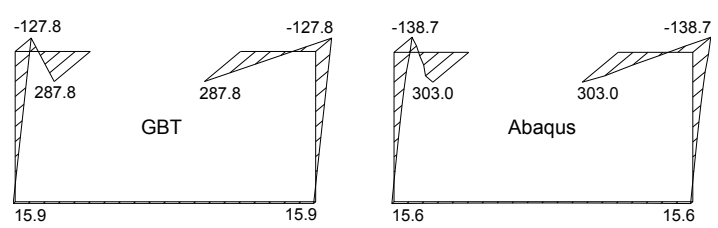

Figure 11: Comparison between the axial normal stress distributions obtained with GBT and Abaqus at the end of the beam. All values are in $\mathrm{MPa}$.

\begin{tabular}{rrrr} 
& GBT [MPa] & Abaqus [MPa] & Difference [\%] \\
\hline$\sigma_{z}$ & 127.7 & 138.7 & 7.9 \\
$\sigma_{s}$ & 197.2 & 196.9 & 0.2 \\
\hline
\end{tabular}

Table 10: Example 2: Stress distributions of GBT and FE analysis.

deviation can be explained by shear lag as the results are here from the end section. The transverse bending stresses at mid-span are shown in Figure 12. Here the
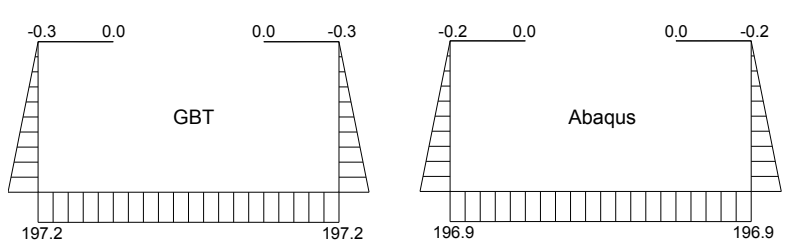

Figure 12: Comparison between the transverse bending stress distributions obtained with GBT and Abaqus at mid-span. All values are in $\mathrm{MPa}$.

maximum deviation is $0.2 \%$ and obtained at the corner in the bottom.

\subsection{Example 3 - Flexural load on box section}

In this third example a box beam is loaded by a flexural load. The geometry, parameters values, discretization of the cross section and the distributed vertical load are as given and shown in Figure 13. This leads to the

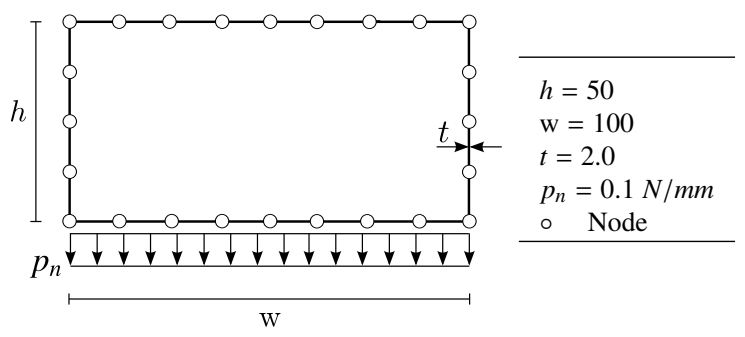

Figure 13: Geometry, parameter values and load for the box beam.

deformation shown in Figure 14. As seen for the lipped channel in example 1, the main deformation is also here related to flexure of the beam. A comparison of the displacement values found using GBT to the FE results found using a Abaqus model is given in Table 11 Here it is seen that the deviation of the maximum displacement in the horizontal direction, $u_{x}$, is $0.0 \%$ and the deviation for the vertical direction, $u_{y}$, is $5.5 \%$. Again the large deviation can be explained by the neglected shear deformations. Calculating the effect of shear deformations as in equation (87) gives a contribution to the displacement of $\Delta u_{y}(z)=0.31 \mathrm{~mm}$ and thus a total displacement 


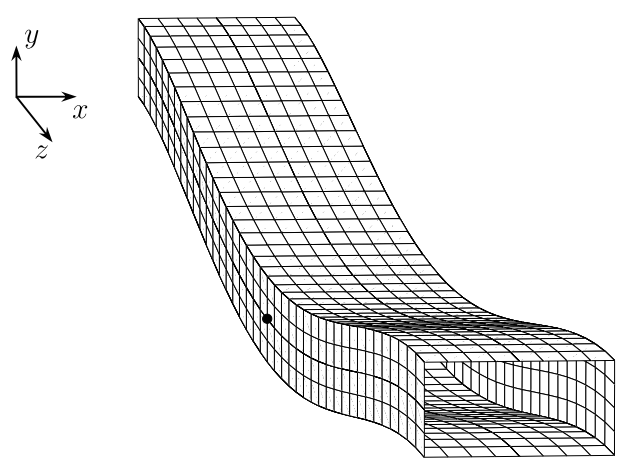

Figure 14: GBT plot of the box beam with a flexural load.

of $u_{y}=6.802 \mathrm{~mm}+0.31 \mathrm{~mm}=7.112 \mathrm{~mm}$. Hereby the deviation is reduced to $1.2 \%$. Making a comparison

\begin{tabular}{rrrr} 
& GBT [mm] & Abaqus [mm] & Difference [\%] \\
\hline$u_{x}$ & 0.056 & 0.056 & 0.0 \\
$u_{y}$ & -6.802 & -7.200 & 5.5 \\
\hline
\end{tabular}

Table 11: Example 3: Nodal displacements of GBT and FE analysis.

between the stress distributions obtained with GBT and Abaqus we obtain the following results. Concerning
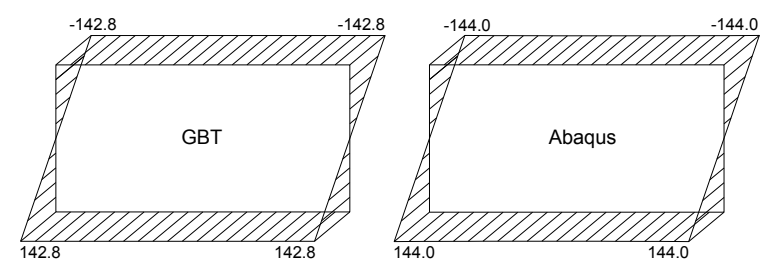

Figure 15: Comparison between the axial membrane stress distributions obtained with GBT and Abaqus for at mid-span. All values are in MPa.
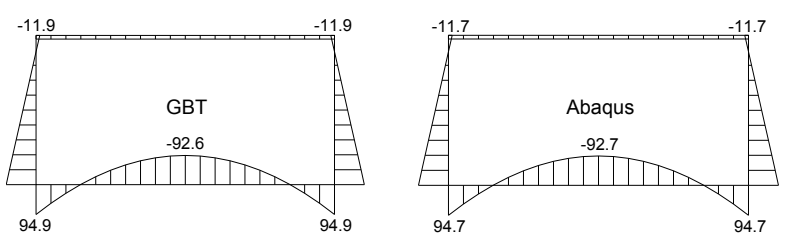

Figure 16: Comparison between the transverse bending stress distributions obtained with GBT and Abaqus at mid-span. All values are in $\mathrm{MPa}$

the axial membrane stresses in the $z$ direction at midspan we obtain the values and distribution presented in Figure 15. In this case a maximum deviation of $0.8 \%$ is obtained as given in Table 12. The transverse bending stresses at mid-span are shown in Figure 16 and shows a maximum deviation of $1.7 \%$.

\begin{tabular}{rrrr} 
& GBT [MPa] & Abaqus [MPa] & Difference [\%] \\
\hline$\sigma_{z}$ & 142.8 & 144.0 & 0.8 \\
$\sigma_{s}$ & -11.9 & -11.7 & 1.7 \\
\hline
\end{tabular}

Table 12: Example 3:Stress distributions of GBT and FE analysis.

\subsection{Example 4-Distortional load on box section}

In this last example the same box beam as in the previous third example is now loaded by a distortional load as shown in Figure 17. Solving the differential equa-

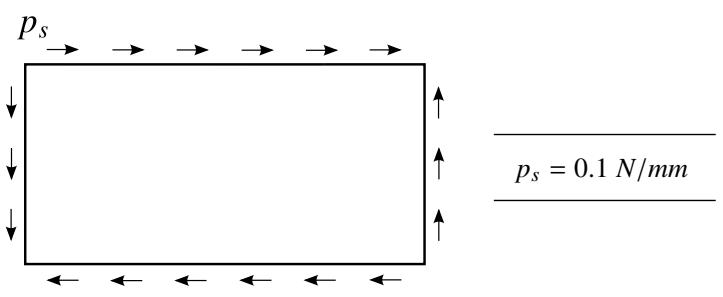

Figure 17: Distributed distortional load.

tions of GBT leads to deformations shown in Figure 18. A comparison of the GBT displacements of the node
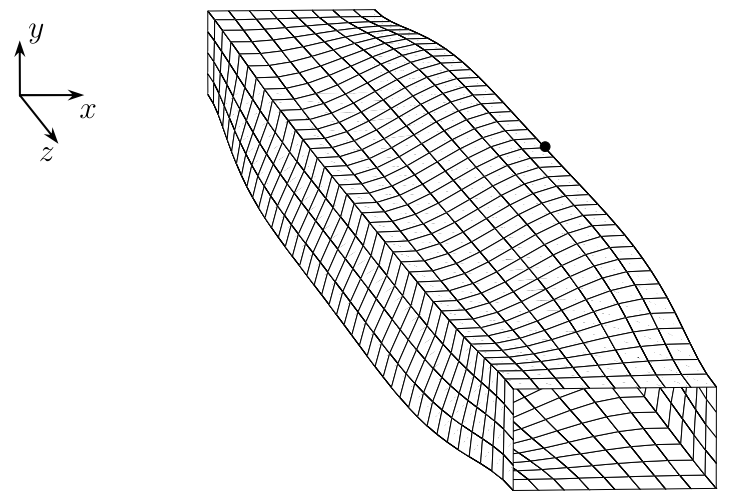

Figure 18: GBT plot of the box beam with a distortional load.

marked in the figure to those found using the Abaqus finite element model, the values is given in in Table 13. Here it is seen that the deviation of the maximum displacement in the horizontal direction, $u_{x}$, is $1.5 \%$ and the deviation for the vertical direction is $1.2 \%$. Having

\begin{tabular}{rrrr} 
& GBT [mm] & Abaqus [mm] & Difference [\%] \\
\hline$u_{x}$ & 0.266 & 0.262 & 1.5 \\
$u_{y}$ & 0.515 & 0.509 & 1.2 \\
\hline
\end{tabular}

Table 13: Example 4: Nodal displacements of GBT and FE analysis. compared the nodal displacement obtained with GBT 
and Abaqus we take a look at the stress distributions. A comparison of the membrane stresses in the $z$ direction at mid-span are shown in Figure 19 which gives a maximum deviation of $2.5 \%$ as shown in Table 14 .
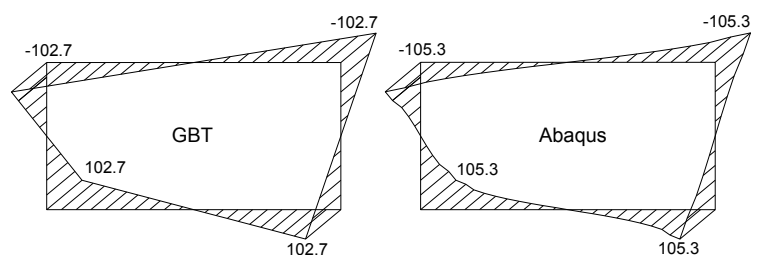

Figure 19: Comparison between the axial normal stress distributions obtained with GBT and Abaqus at mid-span of the beam. All values are in $\mathrm{MPa}$.

\begin{tabular}{rrrr} 
& GBT [MPa] & Abaqus [MPa] & Difference [\%] \\
\hline$\sigma_{z}$ & 102.7 & 105.3 & 2.5 \\
$\sigma_{s}$ & 193.2 & 192.8 & 0.2 \\
\hline
\end{tabular}

Table 14: Example 4: Stress distributions of GBT and FE analysis.

The transverse bending stresses at mid-span are shown in Figure 20. In this case a maximum deviation of 0.2
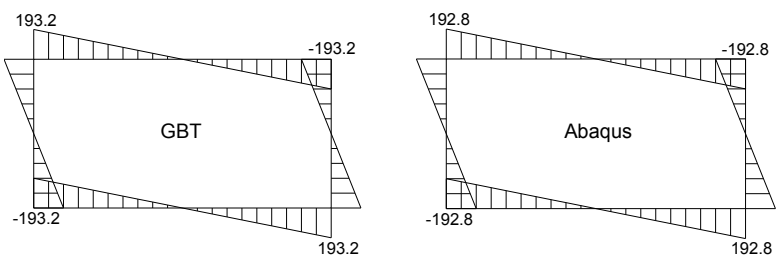

Figure 20: Comparison between the transverse bending stress distributions obtained with GBT and Abaqus at mid-span. All values are in MPa.

$\%$ is obtained.

\section{Conclusion}

In this paper we have included distributed loads in a novel semi-discretized formulation of the distortional differential equations. By using the distortional modal matrix found for the homogeneous system we have transformed the non-homogeneous distortional differential equations into the eigenmode space, and then obtained the uncoupled set of differential equations including the distributed loads. This uncoupling is very important in GBT, since the shear stiffness contribution cannot be neglected nor approximated by the combination of axial stiffness and transverse stiffness, especially for closed cross sections. This means that conventional modal analysis (corresponding to orthogonal damping) cannot be used to solve the equations and analytical solutions must therefore be based on the eigenmodes found for the reduced order distortional differential equations. Examples have been given for thinwalled beams with distributed uniform loads. The chosen examples show solutions which are applicable to finite element formulation of a future distortional beam element with applied loads, i.e. with fixed boundary conditions. The boundary conditions will be handled by the eigenmodes of the homogeneous solution. The examples also show that shear deformation is only included for "Bredt's shear flow" around closed cells. The examples also show that the presented theory does not include shear lag effects. However these and other effects may be included as extensions in approximate energy based finite element formulations which may be used to extend the capabilities of beam elements. The novel approach presented in this paper is a considerable theoretical achievement, since it without approximation gives the full analytical solution of the GBT equations with distributed loads for a given discretization of the cross section.

\section{References}

[1] J. Jönsson, and M. J. Andreassen, Distortional eigenmodes and homogeneous solutions of semi-discretized thin-walled beams. Thin-Walled Structures, 49, 691-707, 2011.

[2] R. Schardt, Eine Erweiterung der technischen Biegelehre für die Berechnung biegesteifer prismatischer Faltwerke. Der Stahlbau, 35, 161-171, 1966.

[3] R. Schardt, Verallgemeinerte Technische Biegetheorie. SpringerVerlag, Germany, 1989.

[4] J.M. Davies and P. Leach, First-order generalised beam theory. J. Construct. Steel Research, 31(2-3), 187-220, 1994.

[5] J. Lepistö and S. Nikula and E. Niemi, Optimum design of coldformed sections using Generalized Beam Theory. Proceedings of the Second International Conference on Coupled Instabilities in Metal Structures, (CIMS 1996 Liége, 05-07/09), J. Rondal and D. Dubina and V. Gioncu (eds.), Imperial College Press, London, 101-108.

[6] S. Rendek and I. Baláž, Distortion of thin-walled beams. ThinWalled Structures, 42, 255-277, 2004.

[7] P. Simão and L. Simões da Silva, Comparative analysis of the stability of open and closed thin-walled section members in the framework of Generalized Beam Theory. Proceedings of the 3rd European Conference on Steel Structures, (EUROSTEEL 2002 Coimbra, Portugal, 19-20/09), A. Lamas and L. Simões da Silva (eds.), 711-721 (vol. 1).

[8] R. Gonalves, P.B. Dinis and D. Camotim, GBT formulation to analyse the first-order and buckling behaviour of thin-walled members with arbitrary cross-sections. Thin-Walled Structures, 47, 583-600, 2009.

[9] R. Gonalves and D. Camotim, Steel-concrete composite bridge analysis using generalised beam theory. STEEL AND COMPOSITE STRUCTURES, 10, 223-243, 2010.

[10] N. Silvestre and D. Camotim, First-order generalised beam theory for arbitrary orthotropic materials. Thin-Walled Structures, 40, 755-789, 2002. 
[11] N. Silvestre, Generalized Beam Theory: New formulations, Numerical Implementation and Applications. Ph.D. Thesis, IST Technical University of Lisbon, Portugal, 2005. (in Portuguese).

[12] M. Hanf, Die geschlossene Lösung der linearen Differentialgleichungssysteme der Verallgemeinerten Technischen Biegetheorie mit einer Anwendung auf die Ermittlung plastischer Grenzlasten. Instituts für Werkstoffe und Mechanik im Bauwesen der TU Darmstadt, 9, 1989.

[13] J. Jönsson, Distortional theory of thin-walled beams. ThinWalled Structures, 33, 269-303, 1999.

[14] C.F. Kollbrunner and N. Hajdin, Dünnwandige Stäbe 1, Stäbe mit undeformierbaren Querschnitten. Springer-Verlag, Berlin, 1972, 1975. 